Christoph Meinel

\title{
Zur Sozialgeschichte des chemischen Hochschulfaches im 18. Jahrhundert*
}

Summary: The social history of chemistry as an academic discipline is dealt with in terms of competing programs of research and institutionalization, focussing on chemistry's transition from a medical curriculum to an economic and scientific context, as exemplified by universities such as Greifswald, Halle, Göttingen, and Jena in the late eighteenth century. Special attention is given to those factors that constitute, stabilize, and, eventually, re-formulate disciplinary identity.

Schlüsselwörter: Chemie, Disziplingeschichte, Sozialgeschichte; Universitäten: Greifswald, Halle, Göttingen, Jena; XVIII Jh.

Sozialgeschichte der Wissenschaften zielt auf zwei scheinbar klar unterschiedene Seinsbereiche: erstens auf die Wissenschaft selbst als kollektive kulturelle Aktivität, die kognitive Elemente (Kenntnisse, Ordnungskriterien und deren Verknüpfungsanweisungen) mit konkreten Handlungsvorgaben verbindet, wobei letztere sowohl den kognitiven Bereich (Kenntnisvermehrung, Systematisierung/Formalisierung, Theoriebildung), als auch den Bereich technisch-praktischer Anwendungen betreffen können; zweitens auf den allgemeingesellschaftlichen (politischen, ökonomischen und sozialen) Kontext einschließlich seiner sozialpsychologischen Prämissen.

$\mathrm{Da}$ Wissenschaft und zumal Naturwissenschaft ihrem Wesen nach aber auf Veränderung angelegt ist und in den gesellschaftlichen Kontext, innerhalb dessen sie sich entfaltet, verändernd eingreift, revidiert sie die Voraussetzungen ihrer eigenen Existenz und die Bedingungen ihrer Fortentwicklung immer wieder von neuem. Einer Sozialgeschichte der Wissenschaft kann es deshalb nicht allein darum gehen, gesellschaftliche "Hintergründe“ zu erhellen, um auf diese Weise dem eigentlichen Gegenstand, der Wissenschaft selbst, schärfere Konturen zu verleihen. Die oft genug vorgenommene Dreiteilung in „Voraussetzungen“, „Wissenschaftsentwicklung selbst“ und „Auswirkungen“ verkennt die zum Wesen von Wissenschaft gehörende Interdependenz dieser drei Ebenen und führt daher bloß wieder auf die alte, untaugliche Dichotomie in ,interne" und "externe“ Faktoren der Wissenschaftsentwicklung zurück. An die hermeneutischen Möglichkeiten einer integrativen Sozialgeschichte der Wissenschaft reicht eine solche Betrachtungsweise nicht heran.

Sozialgeschichte der Wissenschaft ${ }^{1}$ hat die Funktion, die für Wissenschaft charakteristischen Wechselwirkungen kognitiver und sozialer Prozesse zu erforschen, und zwar sowoht im historischen Detail als auch hinsichtlich ihrer strukturellen Entsprechungen².

* Erweiterte Fassung eines Vortrages, gehalten auf dem XXIV. Symposion der Gesellschaft für Wissenschaftsgeschichte, „Sozialgeschichte der Wissenschaften“, 8.-10. Mai 1986 in Lübeck. 
Sie berührt sich darin mit den Aufgabenfeldern der historischen Wissens- und Wissenschaftssoziologie, der Sozialgeschichte der Wissenschaftler, der Berufs- oder Standesgeschichte, der Institutionengeschichte und der Mentalitätsgeschichte. Sie unterscheidet sich von diesen darin, daß es ihr vorrangig darum geht, soziale und sozialpsychologische Veränderungen mit entsprechenden Veränderungen in Gehalt und Struktur der Wissenschaft ursächlich zu korrelieren.

Diese These am Beispiel der Chemie, noch dazu der Chemie des 18. Jahrhunderts, exemplifizieren zu wollen, mag problematisch erscheinen. Sozialgeschichtliche Untersuchungen zur Chemiegeschichte liegen erst in geringer Zahl vor ${ }^{3}$; wirkliche Detailstudien, auf die Sozialgeschichte nie verzichten kann, fehlen, von biographischen Arbeiten einmal abgesehen, fast gänzlich ${ }^{4}$; weite, für die Sozialgeschichte der Wissenschaft zentrale Quellengruppen - erwähnt seien hier bloß Lehrbücher ${ }^{5}$ und Fachzeitschriften sind bisher erst in Ansätzen erfaßt und ausgewertet. Die historische Wissenschaftssoziologie hat sich deshalb mit gutem Grund auf den verläßlicheren Boden des modernen, schärfer abgrenzbaren Wissenschaftsbegriffes, vorzugsweise auf den der exakten Naturwissenschaften beschränkt. Von da aus gesehen, verdient die Chemie des 18. Jahrhunderts kaum die Bezeichnung einer Naturwissenschaft ${ }^{6}$. Ihr Hauptmerkmal ist das der Inhomogenität und Inkohärenz in theoretischer wie in sozialer Hinsicht. Wüßten wir nicht, was Chemie ist, so würden wir die unterschiedlichen „Chemien“ jener Zeit wohl kaum als zusammengehörig empfinden. Chemiatrie und Metallurgie, korpuskularchemische und dynamische Auffassungen, Pflanzenchemie und Phlogistik standen nur lose verbunden nebeneinander. Apotheker und Probierer, Mineralogen und Ärzte setzten chemische Kenntnisse praktisch ein, gehörten aber doch ganz verschiedenen sozialen und intellektuellen Umgebungen an. Ebenso weit spannt sich das Spektrum der Institutionen: Universität und Apotheke, Probierkammer und Akademielaboratorium, Medizinische und Philosophische Fakultät boten vollkommen unterschiedliche Bedingungen für chemische Tätigkeiten. Und doch lassen sich Bereiche ausmachen, in denen sich bereits früh ein Bewußtsein fachlich-wissenschaftlicher Identität und Eigenständigkeit herausbildete. Es ist dies vor allem der Bereich des chemischen Hochschulfaches ${ }^{7}$, und dies nicht von ungefähr. Die Universität ist der soziale Ort neuzeitlicher Wissenschaft schlechthin. Sie ist Schnittstelle des Austauschs zwischen Gesellschaft und Wissenschaft. Als Organisations- und Institutionalisierungsform komplexer intellektueller Arbeit, die ihr Zentrum im Wissensaustausch und der Wissensübermittlung hat, übernimmt die Hochschule für Gesellschaft und Wissenschaft zentrale Funktionen: Qualifikation und Selektion, Innovation und Stabilisierung, Tradition und Legitimation ${ }^{8}$. Diese Funktionen werden im wesentlichen von den wissenschaftlichen Disziplinen, den eigentlichen Differenzierungsformen neuzeitlicher Wissenschaft, wahrgenommen ${ }^{9}$. Disziplingeschichtliche Fragestellungen zählen daher zum Kernbereich einer Sozialgeschichte der Wissenschaft.

Disziplingeschichte - diese Einschränkung ist notwendig - erfaßt freilich immer nur einen bestimmten Ausschnitt der Wissenschaftsgeschichte und damit auch bloß einen Ausschnitt der Sozialgeschichte der Wissenschaft. Sie vernachlässigt systematisch all diejenigen Bereiche, in denen sich Wissenschaft anders als über die Aufgabe gelehrter Wissensübermittlung und intellektueller Sozialisation in eine Scientific Community definiert. Doch da der Wissenschaftsbegriff selbst zunehmend - und gewiß nicht ohne Zutun derer, die ihn jeweils prägen - von denjenigen Formen von Wissenschaft in Anspruch genommen wird, die sich auf Hochschulen und in anderen gelehrten Institutionen durchgesetzt haben, erfaßt die Disziplingeschichte zugleich den sozialhistorisch signifikantesten 
Teilbereich von Wissenschaftsgeschichte. Dies sei zur Rechtfertigung angemerkt, wenn im folgenden unter dem Generalthema einer Sozialgeschichte der Wissenschaft ein disziplingeschichtlicher Ansatz vorgestellt wird.

Wissenschaftliche Disziplinen sind, um eine neuere Definition Robert Kohlers ${ }^{10} \mathrm{zu}$ gebrauchen, gesellschaftliche Institutionen, die akademisches Territorium abgrenzen, Privilegien und Pflichten, die aus Gründen der Fachkompetenz beansprucht werden, zuerkennen, und Ansprüche auf Resourcen legitimieren. Disziplinen bilden gewissermaßen die soziale wie intellektuelle Infrastruktur der Wissenschaft. In Lehrstühlen, Universitätsinstituten, Organisations- und Kommunikationsformen gewinnen sie sichtbare Gestalt. Disziplinen sind historisch gewordene Einheiten und daher Ergebnis kollektiver Anstrengungen, bestimmte Tätigkeits- und Zuständigkeitsfelder zu institutionalisieren. Ihre Grenzen und Strukturen spiegeln menschliche Gewohnheiten und Konventionen wider, nicht etwa eine vorgegebene Naturordnung oder ontologische Kategorien, die im Wesen ihres Gegenstandes lägen. In Disziplinen treffen individuelle und kollektive Wertsysteme (Normvorstellungen einzelner Wissenschaftler, bestimmter Gruppen, der Gesamtheit der Gelehrten oder der Gesellschaft als Ganzer) zusammen und regeln Selbstverständnis, Handlungsnormen und konkretes Verhalten der betreffenden Wissenschaftlergemeinschaft. Die historische Bedeutung von Disziplinen liegt darin, daß diese die genannten Funktionen nicht bloß innerhalb eines gegebenen Zeitraumes, also synchron, ausüben, sondern diachron als wichtigstes traditionsstiftendes Strukturelement fungieren und damit, über die Mechanismen von Wissensvermittlung und Sozialisation, aufeinanderfolgende Wissenschaftlergenerationen verbinden. In dieser spezifischen Funktion hat bereits das Mittelalter die Disziplin von der Wissenschaft im allgemeinen unterschieden ${ }^{11}$. Auch der ursprüngliche Wortsinn von disciplina weist auf die Funktion des schulmäßig Ordnenden, aber auch des sozial wie intellektuell Disziplinierenden hin.

Disziplingeschichte hat gegenwärtig Konjunktur; ihre methodologischen und systematischen Konturen sind freilich noch kontrovers. Während in der wissenschaftshistorischen Forschung der DDR im Anschluß an Thesen, die Martin Guntau ${ }^{12} 1977$ vorgetragen hatte, weitestgehender Konsens ${ }^{13}$ über ein eigenartig statisches drei- bis vierstufiges Modell der Disziplingenese (Vorgeschichte - Diszplinbildung/Herausbildungsphase - Konsolidierung/Klassiche Phase - Moderne Phase) zustandegekommen ist, sind von der Wissenschaftsgeschichtsschreibung und historischen Wissenschaftssoziologie des Westens verschiedene Modelle herausgearbeitet worden, unter denen hier lediglich auf die Arbeiten von Thomas S. Kuhn ${ }^{14}$, Joseph Ben-David ${ }^{15}$, Frank R. Pfetsch und Avraham Zloczower ${ }^{16}$ sowie Peter Weingart ${ }^{17}$ verwiesen sei. Neuerdings hat Rudolf Stichweh ${ }^{18}$, anknüpfend an Niklas Luhmanns soziologische Theorie der strukturellen Differenzierung ${ }^{19}$, ein auch hinsichtlich seines konkreten historischen Erklärungspotentials interessantes Modell der Ausdifferenzierung und nachfolgenden Innendifferenzierung wissenschaftlicher Disziplinen am Beispiel der Physik vorgestellt. Die meisten dieser Arbeiten und modellmäßigen Beschreibungen legen freilich bewußt oder unbewußt ein gewissermaßen vordarwinistisches Konzept der Disziplingenese zugrunde, wie es der geläufige, in der Wissenschaftsgeschichte aber am Ende irreführende Entwicklungsbegriff impliziert, der ja oft im Sinne einer präformistischen Auffassung verwandt wird, gerade so, als sei das, was sich da im Verlauf der Geschichte entwickelt hat, der Sache nach bereits von Anfang an vorhanden gewesen und bedürfe nur mehr einer allmählichen Befreiung von äußerlichem Hüllwerk ${ }^{20}$.

Der Disziplingeschichte wie auch der Sozialgeschichte der Wissenschaft allgemein wäre stattdessen eine Betrachtungsweise adäquat, die gewissermaßen epigenetisch historische 
Wandlungsprozesse verfolgt und damit auch substantielle Transformationen des Wissenschafts- und Disziplinbegriffs einschließlich seiner inhaltlichen und sozialen Merkmale zuläßt. Es wäre daher angemessener, statt von der Entwicklung einer Wissenschaft vom Disziplin- oder Wissenschaftswandel zu sprechen ${ }^{21}$.

Wie eine solche Disziplingeschichte aussehen könnte, hat unlängst Robert Kohler ${ }^{22}$ am Beispiel der biochemischen Disziplinen vorgeführt. Bezeichnenderweise beruft Kohler sich dabei auf die von Charles Rosenberg geprägte biologistische Metapher einer „ecology of knowledge“ ${ }^{23}$ und begreift die Geschichte einer wissenschaftlichen Disziplin als das Ergebnis eines kollektiven, kompetitiven Prozesses, der unter Adaptation an die sich wandelnden sozioökonomischen Verhältnisse seiner Umgebung neue soziale Strukturen schafft und diese institutionell stabilisiert. Dahinter steht die Beobachtung, daß wissenschaftliche Disziplinen, zumal in der turbulenten Phase ihrer Entstehung, alles andere als in sich homogene Einheiten sind ${ }^{24}$. Sie setzen sich vielmehr aus unterschiedlichen Gruppen und Individuen zusammen, deren Forschungsprogramme, Handlungsnormen und Institutionalisierungsstrategien miteinander konkurrieren. Die Divergenz der Programme und Stile erklärt sich aus der Anpassung an das jeweilige soziale und institutionelle Ambiente. Erfolg oder Mißerfolg hängen dabei wesentlich vom Grad der Übereinstimmung von disziplinärem Programm und historischen Realisierungsmöglichkeiten ab. Disziplinwandel resultiert mithin aus vielschichtig rückgekoppelten Prozessen intellektueller, sozialer und ökonomischer Konkurrenz und Adaptation. Der Gesamtpozeß der Genese einer wissenschaftlichen Disziplin ist daher nicht als lineare Abfolge einzelner, auf ein und dasselbe Ziel gerichteter Entwicklungschritte zu sehen, sondern als ein mehrstufiger Vorgang, dessen einzelne Stränge durchaus unterschiedlichen Institutionalisierungszielen zustrebten.

Der geschilderte mehrphasige Verlauf läßt sich in der Geschichte des chemischen Hochschulfaches während des 18 . Jahrhunderts deutlich verfolgen ${ }^{25}$. Im Rahmen der vorliegenden Arbeit kann dieser Vorgang jedoch nur in seinen wichtigsten Etappen skizziert werden. Anschließend soll diejenige Phase, in der die Chemie sich aus ihrer traditionellen Anbindung an die Medizinische Fakultät löst und in die Philosophische Fakultät übertritt, wegen ihrer herausragenden Bedeutung für die Disziplin näher beleuchtet und an einzelnen signifikanten Beispielen konkretisiert werden.

Nachdem die Chemie zunächst in der zweiten Hälfte des 17. Jahrhunderts unter den Prämissen der paracelsistischen Wissenschaftsreform ${ }^{26}$ an den Universitäten hatte Fuß fassen können, ist sie hier bald - unter Abkehr vom ursprünglichen chemisch-kosmologischen Programm - als Hilfswissenschaft mit praktisch-pharmazeutischer Aufgabenstellung in den Lehrplan der Medizinischen Fakultäten inkorporiert worden. Damit war ein außerordentlich wichtiger Schritt vollzogen, und zwar sowohl was den Gegenstand, als auch was die soziale und akademische Stellung des Faches angeht. Denn die Eingliederung in die Medizinische Fakultät bedeutete nicht nur eine Befreiung aus dem Verdacht alchemischer Obskurität, sondern führte auch aus dem starren Theorierahmen der neuaristotelischen oder physikalisch-mechanistischen Naturphilosophie heraus. Damit erhielt das Fach in sachlicher wie in didaktischer Hinsicht ein neues Profil. Darüberhinaus war mit der Aufnahme in eine der „höheren“ Fakultäten ein Zuwachs an akademischem und sozialem Prestige einschließlich einer wirtschaftlichen Besserstellung verbunden; denn noch immer galt die Philosophische oder auch Artistenfakultät, wie sie häufig genannt wurde, 
als bloße Vorstufe für die drei oberen, den Brotwissenschaften vorbehaltenen Fakultäten, und entsprechend war es um die Stellung ihres Lehrkörpers bestellt. Freilich leitete sich das damit hinzugewonnene gesellschaftliche Ansehen weniger von der Stellung als Chemiker her, sondern profitierte vor allem vom hohen Rang des ärztlichen Standes. Schließlich - und dieser Aspekt dürfte für die kognitive Entwicklung des Faches der wichtigste gewesen sein - setzte die Hinwendung zu konkreten Aufgaben im pharmazeutischen Bereich die Chemie der Forderung aus, sich praktisch bewähren müssen.

Die Einbindung in die Medizinische Fakultät hatte für die fachliche Weiterentwicklung der Chemie jedoch noch eine andere und auf lange Sicht eher hinderliche Konsequenz. $\mathrm{Da}$ sie hier nämlich als Hilfswissenschaft in der Regel vom rangniedrigsten Professor meist in Verbindung mit Anatomie, Botanik oder Arzneimittellehre - vorgetragen wurde, war damit auch ihre niedrige Stellung innerhalb der institutionellen Hierarchie strukturell festgeschrieben, ein Dilemma, das bemerkenswerterweise Johannes Hartmann, mit dem die Geschichte des chemischen Hochschulfaches beginnt, bereits im Jahre 1609 klar vorausgesehen hatte ${ }^{27}$. Solange die Chemie aber allgemein in die niedrigste Gruppe der medizinischen Lehrfächer eingestuft wurde, war der Weg zur fachlichen Emanzipation blockiert; denn Fachprofessuren im eigentlichen Sinn waren unbekannt. Oft waren die Lehrstühle lediglich in solche für die mehr theoretischen und solche für die mehr praktischen Fächer unterteilt; in erster Linie handelte es sich dabei jedoch um eine Abstufung nach Rang und Besoldung ohne Rücksicht auf das jeweilige Lehrgebiet. Anstelle einer strengen fachlichen Festlegung der einzelnen Professuren gab es in jeder Fakultät eine feststehende Rangfolge des „Aufrückens“, die im wesentlichen durch Anciennität bestimmt wurde ${ }^{28}$. Die Folge war, daß der Vertreter der Hilfswissenschaften und der propädeutischen Fächer, sobald sich Gelegenheit bot, in die nächsthöhere Position aufrückte, um schließlich die eines Professor primarius einzunehmen, der sein Lehramt oft mit einer einträglichen ärztlichen Privatpraxis zu verbinden wußte. Dieses System des Aufrückens, ein Erbe der mittelalterlichen Universität, war zwar schon früh als unzulänglich erkannt worden, hielt sich aber dennoch bis weit ins 18. Jahrhundert hinein. Die Folge war, daß es sich ein Mediziner kaum leisten konnte, seinen beruflichen Ehrgeiz ganz auf eine der Hilfswissenschaften zu verlegen, ohne damit zugleich seine spätere akademische Karriere zu gefährden. So wurde das von den Medizinprofessoren oftmals stiefmütterlich behandelte Fach zum Betätigungsfeld von außeruniversitären Lehrern ${ }^{29}$, Doctores legentes oder Fakultäts-Assessoren, die auf diesem Wege den Einstieg in eine akademische Position zu schaffen hofften ${ }^{30}$.

Aus diesem strukturellen Dilemma des jungen Faches heraus entstanden nun zu Beginn des 18. Jahrhunderts zwei unterschiedliche Argumentations- und Institutionalisierungsstränge. Beiden ging es darum, die Chemie aus ihrer engen, hilfswissenschaftlichen Anbindung an die Medizin zu befreien, weil diese der Autonomie und disziplinären Differenzierung des eigenen Faches, damit aber auch den Karrierevorstellungen seiner Vertreter entgegenstand. Es ging darum, der Chemie ein neues fachliches Selbstverständnis zu geben ${ }^{31}$. Zwei Richtungen konkurrierten hierbei miteinander um inhaltliche und institutionelle Vorteile: Die erste Richtung, die sich von der Leidener iatromechanischen Schule herleitete, suchte, von einer korpuskular begründeten Chemie ausgehend, eine rationale Physiologie und Pathologie zu entwerfen; die zweite Richtung, die stärker der physikalischen, Newtonisch-Cartesischen Tradition verpflichtet war, war hingegen darauf aus, eine auf Korpuskeln und wirkenden Kräften beruhende allgemeine Wissenschaft von der Natur stofflich-chemisch zu begründen ${ }^{32}$. Ich habe diesen Vorgang die programmati- 
sche Umwertung von der Hilfswissenschaft zur Grundlagenwissenschaft genannt ${ }^{33}$ und messe ihm disziplingeschichtlich insofern Bedeutung bei, als er nicht nur alternative Forschungsprogramme, sondern zugleich mögliche Institutionalisierungswege der Chemie vorzeichnete. An den Universitäten hat sich freilich nur die erste Richtung durchsetzen können; denn nur die Medizinischen Fakultäten und die Funktion der Ausbildung von Ärzten kamen als Basis für die Etablierung eines selbständigen Hochschulfaches Chemie infrage. Doch auch hier setzten die geringen Studentenzahlen (ein bei disziplingeschichtlichen Betrachtungen oft unterschätzter Faktor), der Vorrang von Lehr- und Ausbildungsfunktion gegenüber forscherlichen Ambitionen, die hierarchische Struktur der Fakultäten ${ }^{34}$ und der wirtschaftliche Zwang der Zusammenlegung von „Nominalprofessuren“, daß heißt vollamtlich vertretenen Lehrfächern, auf ein und denselben Hochschullehrer den Möglichkeiten einer autonomen fachlichen Entwicklung enge Grenzen. Bis zur Herausbildung chemisch-naturwissenschaftlicher Fachprofessuren innerhalb der Medizinischen Fakultäten war es deshalb noch ein langer Weg. Wichtigste Voraussetzung war die Abkehr vom System des Aufrückens nach Anciennität, dessen Schädlichkeit für die freie Entfaltung der Wissenschaften längst erkannt war. Doch scheiterten Versuche, das Anciennitätsprinzip abzuschaffen, wie sie etwa Gerard van Swieten in den Ländern der Habsburgermonarchie unternahm, immer wieder an der Dauerhaftigkeit der überkommenen Struktur ${ }^{35}$. Nur in den wenigen Fällen, wo die Größe der Universität eine weitergehende Differenzierung zuließ, haben sich in der Zeit zwischen 1750 und 1790 innerhalb der Medizinischen Fakultäten die ersten Fachprofessuren im eigentlichen Sinne entwickeln können, in denen die Chemie meist mit Botanik und/oder Arzneimittellehre verbunden war, ohne daß der Fachvertreter weiterhin rein medizinische Unterrichtsfächer, Anatomie etwa, vortragen mußte. Auf diese Weise eröffneten sich erstmals auch innerhalb der Medizinischen Fakultäten beruflich-forscherliche Möglichkeiten für Wissenschaftler, die nicht nach einer ärztlichen Tätigkeit strebten, sondern ihre Ambitionen allein auf die ehemaligen Hilfswissenschaften richteten.

Parallel zur Ausdifferenzierung der Chemie innerhalb der Medizinischen Fakultäten verläuft nun seit Mitte des 18. Jahrhunderts ein zweiter Institutionalisierungsstrang, der das Fach wieder stärker auf seine gewerblich-ökonomischen Anwendungsbereiche hin orientierte, um es aus seiner institutionellen und sozialen Anbindung an die Medizin herauszuführen. Der schwedische Chemiker Johan Gottschalk Wallerius hat dies auf den Begriff gebracht, indem er die bewußt programmatisch gemeinte Formel von der „angewandten Chemie" in die Debatte warf. Mit Hilfe dieser neuen Wissenschaftskonzeption der Chemie ${ }^{36}$ gelang es, Kenntnisstand und praktische Leistungsfähigkeit des Faches mit dem utilitaristischen Anliegen der Zeit zu verbinden und dem gewandelten Öffentlichkeitsverständnis der Aufklärungswissenschaft ${ }^{37}$ zu entsprechen. Damit war für die weitere Entwicklung des Hochschulfaches ein Orientierungsrahmen vorgezeichnet, der gerade den außermedizinischen, ökonomisch-gesellschaftlichen Bezügen der Chemie eine Schlüsselfunktion zuwies. An den deutschsprachigen Hochschulen, die diesem Vorbild folgten, kam es im letzten Drittel des 18. Jahrhunderts zu einer besonders engen Verbindung von Chemie und Kameralwissenschaften, und zwar nicht nur in personeller und institutioneller Hinsicht, sondern auch, was Arbeitsgebiete und Publikationen selbst angeht. Dabei erfuhr die Chemie seitens der sich gerade erst institutionalisierenden akademischen Kameralistik ${ }^{38}$ wirksame Unterstützung. Für einige Jahrzehnte sollten die „ökonomischen Wissenschaften“, wie sie damals hießen, eine entscheidende Mittlerrolle zwischen akademischer Chemie und staatlicher Öffentlichkeit einnehmen. Fünf Funktionen 
treten dabei besonders hervor ${ }^{39}$ : Die Kameralisten interpretierten die Bedeutung der Chemie für die Gesellschaft; sie ordneten sie den wirtschafts- und ordnungspolitischen Zielsetzungen des Staates unter und legitimierten damit den Anspruch des Faches auf Eigenständigkeit und angemessene Unterstützung; zugleich hoben sie das sozial-ökonomische Veränderungs- und Gestaltungspotential einer chemisch fundierten Stoff- und Verfahrenskunde hervor und verwiesen die Gesellschaft damit auf ihr innewohnende, mit Hilfe moderner Naturwissenschaft durchzusetzende Entwicklungs- und Modernisierungsmöglichkeiten. Damit waren entscheidende Voraussetzungen für eine grundsätzliche gesellschaftliche Neubewertung des chemischen Hochschulfaches geschaffen.

Besonders deutlich zeigen sich die institutionellen Folgen dieses Disziplinwandels dort, wo die Chemieprofessur nicht von vornherein auf die Ausbildung künftiger Ärzte zugeschnitten war. Modellcharakter hatten in dieser Hinsicht die Chemielehrstühle der skandinavischen Länder ${ }^{40}$. Nicht unterschätzt werden sollte auch die Rolle der im letzten Jahrhundertdrittel entstandenen Bergakademien ${ }^{41}$ mit Universitätsrang, die ein neues, nicht an der Medizin ausgerichtetes Bild des Chemieprofessors prägten. Hier finden sich denn auch die ersten Fachvertreter, die nie eine medizinische Ausbildung durchlaufen hatten ${ }^{42}$. Die Universitäten haben sich dieser Entwicklung teilweise angeschlossen. Professuren für Chemie in Verbindung mit Ökonomie oder Technologie ${ }^{43}$ sind eine charakteristische Erscheinung der Zeit zwischen 1775 und 1820. Oft waren diese eigenen Kameralfakultäten oder fakultätsübergreifenden staatswirtschaftlichen Instituten zugewiesen ${ }^{44}$. Als Hochschulfach hatte die Chemie damit ein Terrain hinzugewonnen, auf dem sie sich weder gegen die Ausbildungsinteressen der Medizinischen Fakultät zu behaupten brauchte, noch dem Vorwurf des bloß Handwerklichen oder Unwissenschaftlichen ausgesetzt war. Die wissenschaftlichen und wirtschaftlichen Erfolge, die die Chemie verhieß, eröffneten ihr zugleich neue Perspektiven der Professionalisierung und fachlichen Institutionalisierung. Auch sollte in diesem Zusammenhang nicht übersehen werden, daß das Chemiestudium im Rahmen der Kameralia Bevölkerungsschichten zugänglich geworden war, die zuvor kaum Zugang zu einer akademischen Ausbildung gehabt hatten. Dies trifft nicht nur für die seit den 1790er Jahren rasch wachsende Gruppe der Apotheker zu, sondern aus zeitgenössischen Berichten wissen wir, daß darüberhinaus eine nicht unbeträchtliche Zahl von Fabrikanten, Gewerbetreibenden, Materialienhändlern und Verwaltungsbeamten chemische Vorlesungen hörten.

Faßt man diese unterschiedlichen Institutionalisierungstendenzen zusammen, so läßt sich sagen, daß in der Chemie seit der Mitte des 18. Jahrhunderts im wesentlichen drei disziplinäre Programme um akademisches Territorium konkurrierten: Einerseits der Zug zur Einrichtung chemischer oder chemisch-botanischer Fachprofessuren innerhalb der Medizinischen Fakultäten, wo die Chemie unter Einschluß der Pharmazie zwar überwiegend auf die Funktion der Medizinerausbildung beschränkt blieb, ansonsten aber weitgehende fachlich-soziale Autonomie genoß: Weder war ihr Unterricht mit dem in rein medizinischen Lehrfächern verbunden, noch brauchte der Fachvertreter im Laufe seiner akademischen Tätigkeit nach „höheren“ medizinischen Professuren zu schielen.

Andererseits erwies sich die Anbindung an die Ausbildungsfunktion der Kameralwissenschaften für den Bedarf der staatlichen Verwaltung als erfolgreicher Weg, die Chemie aus dem medizinischen Kontext zu lösen und ihr einen neuen Ort im sozialen wie akademischen Gefüge zuzuweisen. Hier konnte sie ihre Leistungsfähigkeit sogar besser unter Beweis stellen, als dies angesichts des damaligen Theorie- und Kenntnisstandes auf medizinisch-pharmazeutischem Gebiet möglich gewesen wäre. Obwohl das Beckmann- 
sche Programm einer akademischen Technologie letztlich scheiterte und die alten Kameralwissenschaften im ersten Drittel des 19. Jahrhunderts auseinanderbrachen, so daß sich die institutionelle Verbindung von Chemie, Technologie und Kameralistik wieder löste, sollte die Bedeutung dieses Zwischenspiels nicht unterschätzt werden. Denn als in den 1790er Jahren die neue antiphlogistische Chemie, die sich - wie ein Blick auf die paradigmatischen Arbeiten Lavoisiers belegt ${ }^{45}$ - von Anfang an als naturwissenschaftliche, nichtmedizinische Disziplin verstand, in Deutschland ihren Siegeszug antrat, war der konzeptionelle und institutionelle Rahmen für eine Entwicklung der Chemie außerhalb des medizinischen Curriculums bereits vorhanden.

Als dritter Institutionalisierungsweg zeichnet sich ebenfalls noch im letzten Jahrhundertdrittel ein Prozeß ab, der die Chemie als allgemeine, auf theoretischen Erkenntnisfortschritt zielende Naturwissenschaft in die Philosophische Fakultät als den eigentlichen Ort der modernen, forschungsorientierten Wissenschaft hinüberführen sollte. Doch sind die frühesten Versuche, chemischen Unterricht im Lehrplan der Philosophischen Fakultät unterzubringen, selten von Erfolg gewesen. Noch immer verstand sich die untere Fakultät überwiegend als bloße Vorbereitungsstufe für die höheren, auf bestimmte Berufe hinführenden „Fakultätswissenschaften“. Ein Fach wie die Chemie schien hier fehl am Platze. Auch wenn ihr die Verbindung mit dem kameralwissenschaftlichen und technologischen Unterricht für begrenzte Zeit einen tragfähigen, von traditionellen sozial-hierarchischen Bindungen weitgehend unabhängigen Institutionalisierungsweg eröffnet hatte, so bedurfte es doch eines zusätzlichen Anstoßes, um dieser Richtung zum Durchbruch zu verhelfen: Genau dies ist der Punkt, an dem die Pharmazie für die Geschichte der chemischen Hochschuldisziplin historische Bedeutung erlangen sollte. In dem Moment, in dem sich das Programm einer ökonomisch-gewerblich orientierten Chemie erschöpft hatte, die Philosophische Fakultät aber noch keine ausreichende Basis für die Etablierung einer chemischen Naturwissenschaft abgeben konnte, entwickelte sich mit der aus Reformbestrebungen innerhalb der Apothekerschaft hervorgegangenen und seit 1804 auch legislatorisch sanktionierten ${ }^{46}$ Aufgabe der Apothekerausbildung ein neues, von Chemikern wie Liebig geschickt benutztes Institutionalisierungskonzept, das - parallel zum Funktionsund Strukturwandel der Philosophischen Fakultät allgemein ${ }^{47}$ - letztlich in die Hochschulchemie des 19. Jahrhunderts überleitete. Dieser Schritt ist gerade auch unter sozialgeschichtlichem Aspekt recht gut untersucht ${ }^{48}$, führt aber über den zeitlichen Rahmen der vorliegenden Betrachtungen hinaus.

Um das geschilderte Modell des Disziplinwandels der Chemie historisch zu konkretisieren, soll im folgenden die entscheidende Phase der gleichzeitigen Konkurrenz dreier divergierender Institutionalisierungsprogramme (chemische Fachprofessur in der Medizinischen Fakultät - kameralwissenschaftlich orientierte Chemie - Chemie als Naturwissenschaft in der Philosophischen Fakultät) am Beispiel von vier ausgewählten Hochschulen näher vorgestellt werden.

Wenn wir uns dafür zunächst die kleine und wissenschaftlich nicht eben sonderlich markante pommersche Universität Greifswald vornehmen, so nicht allein deshalb, weil die Verhältnisse hier relativ gut untersucht $\operatorname{sind}^{49}$, sondern auch, weil Greifswald, das bis 1815 zum schwedischen Reichsgebiet gehörte, nicht nur für den schwedisch-deutschen Wissenschaftstransfer allgemein ${ }^{50}$, sondern auch für die Einführung der Walleriusschen 
Konzeption „angewandter" Chemie und damit für die ökonomisch-gewerbliche Ausrichtung des chemischen Hochschulfaches in Deutschland von entscheidender Bedeutung war. In der Tat gehörte Greifswald zu den vielen problematischen Kleinstuniversitäten des deutschsprachigen Raumes und ist deshalb nicht untypisch für die Verhältnisse jener Zeit. Denn eine Wissenschafts- und Universitätsgeschichtsschreibung, die bloß die klingenden Namen Halles, Göttingens und dann Berlins im Munde führen wollte, ginge an der sozialen Wirklichkeit von Wissenschaft vorbei. Gegen 1800 zählte Greifswald nur etwa 60 Studenten, 15 Professoren und 5 Adjunkten, und seit mehr als hundert Jahren war hier keine Immatrikulation für Medizin mehr zu verzeichnen gewesen ${ }^{51}$. Versuche, die Attraktivität dieser Fakultät durch Theatrum Anatomicum (1757), Botanischen Garten (1763) oder chemisches Laboratorium ${ }^{52}$ zu steigern, schlugen fehl. Nepotismus, Mißwirtschaft und intellektuelle Selbstgenügsamkeit hatten die Universität in eine Krise gestürzt, aus der sie sich bis zur Mitte des 19. Jahrhunderts nicht recht erholen sollte ${ }^{53}$. Vermutlich traf der schwedisch-pommersche Kammerrat Johann David von Reichenbach den neuralgischen Punkt, wenn er 1786 schrieb $^{54}$ :

Ein anderes wesentliches Gebrechen ist da zunächst: man sieht bey den zu bestellenden Professoren nicht einzig und allein auf Gelehrsamkeit, Ruf und sich ausnehmende Geschicklichkeit. [...] Der junge Mensch reiset im 18. Jahr nach Greifswald, hört hin und wieder bei jemandem, der Einfluß hat, ein Paar Collegia, promoviret, schlägt Stunden ans schwarze Brett, die er nie lieset, hängt sich an ein Mägdchen von Connection, schmeichelt, wartet den Großen auf, wendet hier und dort baare Münze an, und im Umsehen ist er zur allgemeinen Verwunderung Professor.

Das einzige strukturelle Revirement jener Zeit betraf die Philosophische Fakultät, für die der Königlich-schwedische Visitationsrezeß von 1775 unter aufgeklärt-kameralistischer Zielsetzung und in der Absicht, die verheerenden Folgen des Siebenjährigen Krieges in Pommern zu lindern, eine engere Verbindung von Wissenschaft und wirtschaftlicher Praxis vorschrieb und durchsetzte, daß Chemie ${ }^{55}$, Staats-, Ökonomie-, Kameral- und Finanzwissenschaften in den Lehrplan aufgenommen wurden. In diesen Zusammenhang gehört die 1781 erfolgte Ernennung von Bernhard Christian Otto zum Professor für Naturgeschichte und Ökonomie, doch wechselte dieser 1788 als Ordinarius für Naturgeschichte, Botanik und Chemie an die Medizinische Fakultät der Universität Frankfurt/Oder über. Die Universität war der neuen Aufgabe jedoch nicht gewachsen, Schlendrian und Überalterung hatten den Lehrkörper unfähig gemacht, den Herausforderungen der neuen Zeit zu begegnen. Ernst Moritz Arndt, der politische Schriftsteller und Historiker, nach dem die Hochschule noch heute heißt, erinnerte sich an seine eigene Zeit als Fakultätsadjunkt ${ }^{56}$ :

Zweitens war Greifswald eine Versorgungsanstalt für die Söhne und Töchter der Professoren und mancher angesehenen Familien der Stadt. [...] Als ich antrat [1802], waren einige sehr würdige Alte da und etwa ein Dutzend Jüngere. [...] Dies brachte durch das junge Blut etwas Belebung und Erregung in den Greifswalder Schlaf. Es hat sein Mißliches mit solchen Mühlen der Gelehrsamkeit, denen das Wasser, d.h. die Studenten, zu sehr fehlt; es tritt leicht Vertrocknung und Erstarrrung oder Verfaulung ein.

Die herausragende Wissenschaftlerpersönlichkeit Greifswalds zu jener Zeit war ohne Zweifel der vielseitige Christian Ehrenfried Weigel ${ }^{57}$, der an der "modernen“ Universität Göttingen studiert und promoviert hatte, um 1773 als Adjunkt und Inspektor des Botanischen Gartens nach Greifswald zurückzukehren. 1775 zum Professor Chemiae et Pharmaceutices ernannt, steht Weigels wissenschaftlicher Werdegang ganz im Zeichen der neuen, utilitaristischen Auffassung. Seine Antrittsvorlesung mit dem bezeichnenden Titel Der Einfluß Chemischer Kenntisse in die Oekonomie besonders des Schwedischen Pommerns (Greifswald 1775) setzte nicht nur die Richtlinien des unmittelbar zuvor ergangenen Visitationsrezesses in die Praxis des Hochschulunterrichts um, sondern erweist Weigel als en- 
gagierten Fürsprecher einer „ökonomisch“ ausgerichteten Chemie, die vom Staat unter kameralistischen Gesichtspunkten zur gezielten Gewerbeförderung und Erschließung heimischer Rohstoffe eingesetzt werden könne ${ }^{58}$. In Verbindung mit seiner wiederholten Forderung nach Einrichtung eines chemischen Laboratoriums und Ausweitung des chemischen Unterrichts unter geflissentlicher Vermeidung möglicher Interessenkollisionen mit der Medizin zeigt sich hier, daß Weigel ein klares Institutionalisierungsprogramm verfolgte. Dabei übernahm er Begriff und Konzeption der ,angewandten“ Chemie von seinem Landsmann Wallerius, dessen Schriften - wie auch die anderer schwedischer Chemiker - er ins Deutsche übersetzte und somit für die internationale Verbreitung der neuen Konzeption chemischer Wissenschaft sorgte ${ }^{59}$. Der unmittelbare Praxisbezug ist auch für Weigels Unterricht charakteristisch: Neben der Hauptvorlesung „Chemie für Ärzte und Nichtärzte“ las er regelmäßig allgemeine Naturgeschichte, Ökonomie und chemische Technologie (nach den Kompendien von Erxleben und Beckmann, seinen Göttinger Lehrern), medizinische und reine Chemie sowie Mineralogie, und zwar in der Medizinischen, erst nach 1806 gelegentlich auch in der Philosophischen Fakultät. Botanik und Pharmazie traten mehr und mehr in den Hintergrund. Wir haben es hier also mit dem Typus der chemisch-naturwissenschaftlichen Fachprofessur innerhalb der Medizinischen Fakultät zu tun. Obgleich inhaltlich ganz an die ökonomischen Wissenschaften angeschlossen, konnte die Chemie sich wegen der Kleinheit und provinziellen Enge Greifswalds nicht aus der institutionellen Verankerung in der Medizinischen Fakultät lösen. Bezeichnenderweise wurden alle namhaften Schüler Weigels später Mediziner, und erst nach 1826 trat die Chemie hier in die Philosophische Fakultät über.

Nicht uninteressant ist der Vergleich mit Halle. Die 1694 gegründete preußische Renommieruniversität - Zentrum der Aufklärung und ein halbes Jahrhundert später mit 1500 Studenten weit an der Spitze aller deutschen Hochschulen - ist für die Geschichte der Chemie insofern wichtig, als hier kein Geringerer als Georg Ernst Stahl, der Begründer der Phlogistontheorie und Schulhaupt einer ganzen Chemikergeneration, gelehrt hatte und Stahlianer wie der große Systematiker Johann Juncker und dessen Schüler diese Tradition bis über die Mitte des 18. Jahrhunderts hinaus fortführten ${ }^{60}$. Auch institutionell war die Chemie in Halle ungewöhnlich gut gestellt, gehörte sie doch mit Anatomie und Chirurgie statutenmäßig zu den Lehrfächern des Professor primarius practicus, während der an zweiter Stelle rangierenden Professio theoretica die Fächer Physiologie, Pathologie, Hygiene, Materia Medica und Botanik zugewiesen waren ${ }^{61}$. Nicht nur die Rangfolge dieser Aufteilung, sondern auch die Trennung von Chemie und Pharmazie sind bemerkenswert und weisen deutlich auf die Stahlsche Konzeption des chemischen Hochschulfaches.

Eine weitere Besonderheit Halles stellten die zahlreichen Doctores legentes dar, die als Substituenten eines Lehrstuhlinhabers oder als freie Dozenten nicht unerheblich zur Ergänzung und Modernisierung des Lehrangebots beitrugen und darüberhinaus den Professorennachwuchs stellten ${ }^{62}$. Einer von ihnen war Friedrich Albrecht Carl Gren ${ }^{63}$, der zuvor eine Apothekerlehre absolviert und in Erfurt und Helmstedt Medizin und Naturwissenschaften studiert hatte. Seit 1783 in Halle, hat Gren hier zunächst als Adlatus des Professors für Physik und Mathematik Wenceslaus Johann Gustav Karsten, eines entschiedenen Befürworters der Einbeziehung der Chemie in die Naturlehre ${ }^{56}$, physikalisch-chemische Untersuchungen ausgeführt, dann - selbst noch Student - seit 1786 mit Zustimmung der Fakultät chemische und pharmakologische Vorlesungen gehalten. Im Sommer 1786 promovierte er zum Doctor medicinae und - ungewöhlich genug - 
ein halbes Jahr darauf auch noch zum Magister philosophiae ${ }^{65}$, als wolle er sich die Option eines späteren Fakultätswechsels offenhalten. Zur gleichen Zeit erreichte ihn die Ernennung zum unbesoldeten Professor medicinae extraordinarius, zusätzlich kurz darauf die zum Professor physices extraordinarius der Philosophischen Fakultät. Zu Jahresbeginn 1788 wurde bei 150 Talern Gehalt und unter Beibehaltung des medizinischen Extraordinariats die gerade verwaiste ordentliche Professur für Naturwissenschaft in der Philosophischen Fakultät auf ihn übertragen und mit der Direktion des Naturalienkabinetts verbunden. Doch scheint es, als sei diese Entwicklung durchaus nicht im Sinne der Karrierevorstellungen Grens gewesen; denn im August wandte er sich mit einer Eingabe direkt an den König nach Berlin ${ }^{66}$ :

Ich sehe mich aber zugleich durch letztere [die Ernennung zum Ordinarius in der Philosophischen Fakultät] von der medizinischen Facultät ausgeschlossen, worauf ich mir laut meiner Bestallung als extraordinarius Hoffnung machte. Ich verliere nicht allein die Anciennité vor dem Professor Reil, sondern auch überhaupt mehr, als ich in der philosophischen Facultät zu erwarten habe. Ich würde auf diese Art meine Promotion in Doctorem Medicinae als vergeblich anzusehen haben, und nicht mehr mit der Aufmunterung und bessern Aussicht in die Zukunft, die bey einer so mühsamen Stelle, als ich habe, nöthig ist, mich dem Unterrichte unterziehen können. Neben der Naturlehre und Naturgeschichte macht die Chemie, u[nd] die ihr verwandten Wissenschaften, die ich hier einzig lehre, den Gegenstand meines Unterrichtes aus; allein die letztere ist auch von jeher, und auf allen Universitäten zur medizinischen Facultät gerechnet worden.

Der Beschwerde wurde stattgegeben und Gren als ordentlicher Professor loco septimo in die Medizinische Fakultät übernommen, freilich nicht, ohne diese zuvor um Stellungnahme zu bitten, ob sie einen Kollegen, der im Grunde doch Chemiker sei, in ihre Reihen aufnehmen wolle. Die Fakultät befand, daß Gren „ein geschulter Physicus ac Chemicus“ wäre und stellte auch ausdrücklich fest, daß seiner „Decanabilität“ secundum ordinem nichts im Wege stehe, knüpfte den Übertritt aber an die Bedingung, daß er seine Mitgliedschaft in der Philosophischen Fakultät niederlege, weil sich oft Schwierigkeiten ergäben, „wenn ein membrum academicum in zweien Facultaeten Sitz und Stimme hätte“ "67. Auch wenn Gren sich in der neuen Position anfangs bemüßigt fühlte, gelegentlich medizinische oder physiologische Vorlesungen anzuzeigen, so ist er doch Chemiker und Mineraloge geblieben, in der deutschen Auseinandersetzung um Für und Wider der antiphlogistischen Chemie einer der hartnäckigsten Verfechter der alten Auffassung, dessen Harmonisierungsversuche, die vom "negativen Gewicht“ des Phlogistons ausgingen, eine gewisse Bedeutung erlangten ${ }^{68}$. In die gleiche Zeit fällt auch die Gründung seines Journal der Physik (1790-1794, danach 1795-1798 Nenes Journal der Physik), dessen Inhalt und Zielsetzung, ebenso wie ein Ruf an die Bergakademie Freiberg, eindeutig belegen, daß Grens chemische Ambitionen nichts mehr mit Pharmazie oder Medizin zu tun hatten. So war es nur folgerichtig, wenn nach seinem Tod der Bergrat Alexander Nikolaus Scherer ${ }^{69}$, der bei Laurenz Johann Daniel Suckow in Jena allgemeine Naturwissenschaften und Chemie studiert sowie Reisen nach England und zu den Bergbaurevieren Deutschlands angeschlossen hatte, 1799 als Nachfolger Grens berufen wurde und eine ordentliche Professur für Physik und Chemie in der Philosophischen Fakultät erhielt. Ihm folgte mit Ludwig Wilhelm Gilbert 1801 ein im Grunde reiner Physiker ${ }^{70}$. Insgesamt war diese Entwicklung freilich eher untypisch, weil das chemische Hochschulfach hier fast ausschließlich einer in der Person Grens begründeten inneren Entwicklungslogik folgte, die es konsequent aus der medizinisch-pharmazeutischen Aufgabenstellung heraus und in die Richtung einer physikalischen Naturwissenschaft führte, welche sich bloß aus akademisch-hierarchischen und pekuniären Gründen so lange wie möglich des Rückhaltes der Medizinischen Fakultät bediente. Bemerkenswert ist auch, daß konkurrierende disziplinäre Programme in Halle offenbar keine Rolle spielten ${ }^{71}$. 
Gerade dieser letzte Aspekt sollte aber in Göttingen entscheidend werden ${ }^{72}$. Nach dem Vorbild ihrer großen preußischen Rivalin in Halle im Jahre 1734/37 als Universität neuen Typs eröffnet, hatte man sich bewußt vom traditionellen Fächerkanon und der üblichen Rangfolge der Fakultäten abgewandt und auf eine Stärkung der zeitgemäßeren Wissenschaften Wert gelegt ${ }^{73}$. Daß die Chemie dabei nicht fehlen dürfe, verstand sich von selbst ${ }^{74}$. Akademische Lehrfreiheit und der ungehinderte Wettbewerb der Dozenten um die Gunst ihrer Hörer - im ausgehenden 18. Jahrhundert hier und da schon gefordert, in Göttingen aber erstmals verwirklicht - sollten die Attraktivität der Universität heben und die notwendige Flexibilität bei der Berücksichtigung neuer Lehrinhalte gewährleisten. So heißt es in einem um 1770 verfaßten Bericht über die Göttinger Verhältnisse ${ }^{75}$ :

A m besten ist die Universität bestellt, wenn jeder Lehrer sich seine Lieblings- und Hauptwissenschaft selbst erwählt; denn so wird er die Neben- und Hülfswißenschaften nicht auf die Seite setzen. [...] Wo Freyheit zu lesen aufgestellt wird, was jeder will, da wird sich von selbst jeder seine Wißenschaft wählen: Glücklich ist die Universität, wenn in jeder Hauptwißenschaft oder jedem einzelnen Haupttheile derselben wenigsten zwey wichtige Männer als Sterne der ersten Größe am akademischen Himmel glänzen.[...] Eigenes Interesse, Aemulation, Brodneid - alles muß dazu gebraucht werden, die Akademie auf die höchste Stufe des Ruhms zu bringen.

Die Statuten der jungen Universität bestimmten deshalb ausdrücklich, daß jeder Professor das Recht habe, privatim über sämtliche Lehrgebiete seiner Fakultät zu lesen ${ }^{76}$. Die Medizinprofessoren genossen „ex peculiario beneficio Regio“ sogar des Privileg, auch über sämtliche philosophischen Disziplinen lesen zu dürfen. Lediglich die Professoren der Philosophischen Fakultät mußten sich in öffentlichen, daß heißt unentgeltlichen Vorlesungen aus Rücksicht auf die wirtschaftliche Lage ihrer Kollegen auf das Lehrfach ihrer jeweiligen Nominalprofessur beschränken. Das Nebeneinander konkurrierender Dozenten und Fachrichtungen machte sich besonders in der Anfangszeit bemerkbar, als noch nicht alle vorgesehenen Lehrstellen besetzt waren.

Bezeichnend ist hier der Fall des entlaufenen Badenser Pfarrers Johann Christoph Cron, der zuvor ein wenig Medizin studiert hatte und sein geistliches Amt unter spektakulären Umständen aufgab, um 1735 sein Glück an der noch nicht inaugurierten Universität Göttingen zu versuchen, wo er Chemie mit besonderer Rücksicht auf die Mineralogie und Metallurgie des Harzes lehren wollte ${ }^{77}$. Seit 1737 zeigte Johann Andreas Segner, der ordentliche Professor für Physik und Mathematik, dem man nominell auch eine medizinische Professur übertragen hatte, weil noch kein anderer Mediziner zur Hand war ${ }^{78}$, regelmäßig chemische Vorlesungen nach dem Lehrbuch von Teichmeyer an. Auch innerhalb der Naturlehre, über die der Philosophieprofessor Samuel Christian Hollmann von 1734 an las, kamen gelegentlich chemische Fragen vor ${ }^{79}$. Die unübersichtliche Situation des ersten Jahrzehnts stabilisierte sich jedoch bald, nicht zuletzt wegen genauerer Reglementierung der Zulassung zum Lehramt ${ }^{80}$, und die Chemie fand ihren Platz in der Medizinischen Fakultät, freilich nur als Hilfswissenschaft, die man beim Aufrücken in höhere Positionen wieder abgab. So lehnte der Medizinprofessor Johann Gottfried Brendel, der von 1748 an regelmäßig die pharmazeutische Chemie vortrug, eine zusätzliche Ernennung zum Professor Chemiae ab, weil sie mit Verpflichtungen, doch bloß 50 Talern Besoldungszulage verbunden gewesen wäre. Als Brendel dann 1753 zum Lehrstuhl für Anatomie und Chirurgie aufrückte, trat Rudolf Augustin Vogel an seine Stelle. Dieser begann als Extraordinarius, übrigens mit einer chemischen Antrittsvorlesung, und wurde 1760 Professor tertiarius für Medizin und Chemie. Auf chemischem und mineralogischem Gebiet liegen seine bedeutendsten wissenschaftlichen Leistungen, darunter die in vielen Auflagen verbreiteten Institutiones chemiae (Göttingen 1755, deutsch Weimar 1775). Doch 
nachdem Vogel 1773 zum Professor primarius aufgerückt war, widmete er sich nahezu ausschließlich klinisch-medizinischen Fragen.

Das Aufrücken nach Anciennität war zu diesem Zeitpunkt freilich umstritten. Daß sich auch eine Universität wie Göttingen davon nicht freihalten konnte, beweist der Vorfall; doch war man sich der damit verbundenen Probleme bewußt. In einem Gutachten des Hannoveraner Hofrats Johann Daniel Gruber für Gerlach Adolf Frhr. von Münchhausen, den Gründer und ersten Kurator der Universität, heißt es mit warnendem Hinweis auf die Zustände in Jena, jene Hochschule sei ${ }^{81}$

durch die enorme Besetzung der Facultäten mit überflüßigen membris so verhuntzet, als sie niemals gewesen. Philosophi sind Theologi und Medici; Historici sind Juristen. Der Schade besteht darin, daß bey einer Vacanz man die Stelle mit einem andern tüchtigen subjecto zu besetzen keine Freyheit hat, sondern solche Nebenschößlinge in ihrer Ordnung ascendiren laßen muß.

Als es dann darum ging, einen Nachfolger für den im April 1774 gestorbenen Primarius Rudolf Augustin Vogel zu finden, gab auch hier zunächst die Person, nicht das Fachgebiet den Ausschlag. Favorisierter Kandidat war der Duisburger Medizinprofessor Johann Gottlob Leidenfrost, ein Empiriker, der auch naturwissenschaftliche Studien betrieb, doch empfahl der Hannoveraner Geheime Rat dem Ministerium, sich für alle Fälle auch den Tübinger Extraordinarius für Chemie und Botanik, Johann Friedrich Gmelin, für Göttingen zu sichern, „obgleich sich dermalen keine für ihn paßende Lehrstelle erledigt findet" 82 . Weil aber Gmelin in Tübingen bereits ein Gehalt erhielt, mußte man ihm für Göttingen ebenfalls eine Besoldung in Aussicht stellen. Eine reguläre Stelle war nicht verfügbar, und so empfahl der Rat, Gmelin interimistisch „zum Professore philosophiae ordinario und Medicinae extraordinario“ zu bestellen ${ }^{83}$. Dies geschah, nachdem die an einige finanzelle Bedingungen geknüpfte Zusage vorlag ${ }^{84}$, und Ostern 1775 trat Gmelin sein Amt an. Offensichtlich war diese Lösung als Bewährungsprobe gedacht; denn im November 1778 teilte man ihm mit, er werde wegen „bisher rühmlichst bewiesenem Fleiß und nun mehr zu Tage gekommenen Kenntnisse“ zum ordentlichen Professor der Medizin befördert, müsse dafür aber aus der Philosophischen Fakultät ausscheiden ${ }^{85}$. Doch weder auf seine Lehrtätigkeit noch auf seine wissenschaftlichen Arbeiten hat sich dieser Fakultätswechsel ausgewirkt: Gmelin las sommers wie winters Experimentalchemie, dazu alternierend „technische oder auf Handwerker und Künste angewandte Chemie“, „Pharmazie mit Versuchen und Arbeiten“, „Mineralogie“ sowie "Probier- und Schmelzkunst“ ${ }^{86}$, später dann auch „technische, docimastische und metallurgische Chemie mit Experimenten sowie Gelegenheit zu eigenen Arbeiten“, wobei Modelle von Fabriken, Hochöfen und ähnlichem benutzt wurden ${ }^{87}$. Seine Publikationen galten der anorganisch-mineralogischen Chemie, Pharmazie und Metallurgie mit immer stärkerem Einschlag zur Technologie und Gewerbskunde, wobei er der phlogistischen Auffassung treu blieb. Gmelins Professur ist daher als erste echte chemisch-naturwissenschaftliche Fachprofessur innerhalb der Medizinischen Fakultät anzusprechen.

Im unmittelbaren Bezug zur gewerblich-industriellen Praxis, der aus vielen Arbeiten Gmelins spricht, kommt ein Charakteristikum der Göttinger Universität zum Ausdruck: die Bedeutung der ökonomischen und technologischen Fächer. Sie dürfte nicht zuletzt auf den Einfluß Johann Heinrich Gottlob Justis zurückgehen, eines der prominentesten Kameralisten jener Zeit, der von 1755 bis 1757 in Göttingen polizeiwissenschaftliche, ökonomische und mineralogisch-montanwissenschaftliche Vorlesungen gehalten hatte. Justi gehörte nämlich zu denjenigen Vertretern seines Faches, die sich entschieden für die Einbeziehung von Chemie, Bergwissenschaften und Naturlehre in die Kameralistik ein- 
setzten. Auf seinen Rat hin wurde 1758 Christian Wilhelm Büttner als Extraordinarius für Naturgeschichte und Chemie in die Philosophische Fakultät berufen und mit der Ausbildung der Studenten des Kameralfaches betraut. 1763 wurde seine Professur in ein Ordinariat für allgemeine Naturwissenschaften umgewandelt. Weit über Göttingen hinaus reicht die Bedeutung der Professur für ökonomische Wissenschaften, die Johann Beckmann, der Begründer der akademischen Technologie, hier 1766 erhielt und die 1770 in ein Ordinariat aufgewertet wurde. Beckmann bezog Mineralogie, Agrikultur und Warenkunde in den Unterricht ein, er führte seine Studenten auf Exkursionen zu Glashütten und Bergwerken und unterhielt - wenige Jahre nachdem die ersten agrikulturchemischen Lehrbücher ${ }^{88}$ erschienen waren - eine landwirtschaftliche Versuchsstation, seinen „Ökonomischen Garten“. Wenn auch selbst kein Chemiker, so hat Beckmann doch den typischen Anwendungsbereich der frühen, gewerblich-industriellen Chemie zum akademischen Lehrgegenstand erhoben. Aber auch andere Göttinger Professoren haben zumindest zeitweilig chemischen Unterricht erteilt, so der Botaniker Johann Andreas Murray im Rahmen der Arzneimittellehre oder Johann Christian Polykarp Erxleben, der aus der Tierheilkunde (die für gewöhnlich zu den ökonomischen Wissenschaften zählte) herkam, seit 1767 chemische Vorlesungen hielt und 1771 Professor für Physik, Chemie und Naturgeschichte ${ }^{89}$ in der Philosophischen Fakultät wurde. Seine vielbenutzten $A n$. fangsgründe der Chemie (Göttingen 1775) markieren den Beginn einer von Medizin, Pharmazie und Naturgeschichte unabhängigen Tradition deutschsprachiger Chemielehrbücher ${ }^{90}$. In der Vorrede heißt es zur ratio edendi ${ }^{91}$ :

Ein großer Theil unserer chemischen Lehrbücher beschäftigt sich fast mit nichts, als mit der pharmaceutischen Chemie: ich habe die gesammte Chemie abgehandelt, da auch Mancher, der kein Arzt ist oder werden will, ietzt die Chemie studirt, und man einen jeden solchen offenbar zum besten hat, wenn man ihm das Studium der Chemie als so sehr nützlich empfiehlt, und ihm doch hernach unter dem Nahmen der Chemie nichts anders zu lernen gibt, als Pharmacie ${ }^{91}$.

Natürlich darf auch Georg Christoph Lichtenberg nicht unerwähnt bleiben. Zunächst Professor für Mathematik, folgte er 1780 Erxleben auf den Lehrstuhl für Physik und Naturkunde nach und hat hier lebhaften Anteil an der stürmischen Entwicklung der pneumatischen Chemie und der antiphlogistischen Lehre genommen ${ }^{92}$.

Das Göttinger Beispiel macht deutlich, daß innerhalb der Chemie divergierende Institutionalisierungsstrategien, die jeweils spezifische Ausbildungsziele, Forschungsprogramme und Anwendungsbezüge beinhalteten, miteinander um die personellen und finanziellen Resourcen der Universität konkurrierten.

Jena, eine Hochburg der Aufklärung und mit 700-800 Studenten nach Halle und Göttingen die drittgrößte deutsche Universität, war die erste, an der sich die institutionelle Trennung von Chemie und Medizin auf Dauer durchsetzen konnte. Der Grund dürfte darin zu suchen sein, daß es hier neben der pharmazeutisch-medizinischen Chemie bereits seit langem chemischen Unterricht in der Philosophischen Fakultät und eine einflußreiche akademische Kameralistik gegeben hatte ${ }^{93}$. In der Medizinischen Fakultät reicht die Tradition der Chemie nahezu ununterbrochen bis ins frühe 17. Jahrhundert zurück ${ }^{94}$. Bedeutende Iatrochemiker wie Werner Rolfinck, Georg Wolfgang Wedel oder der als chemischer Lehrbuchautor geschätzte Hermann Friedrich Teichmeyer ${ }^{95}$ beweisen, daß sich in Jena immer wieder wissenschaftlich hervorragende Medizinprofessoren der Chemie annahmen. Dies erklärt sich nicht zuletzt daraus, daß die Chemie in Jena seit 1641 - wie ein halbes Jahrhundert später auch in Halle - zu den Lehrfächern der ranghöchsten Professur, der Professio chymiae et praxeos, zählte. In der zweiten Hälfte des 
18. Jahrhunderts verblaßte dieser Glanz aber aufgrund der finanziellen Krise der Hochschule und weil in Halle, besonders aber in Göttingen ernstliche Konkurrenz entstanden war. Dafür entwickelte sich Jena zu einem wichtigen Zentrum der Kameralwissenschaften, seitdem hier mit Joachim Georg Darjes von 1744 bis 1763 einer der einflußreichsten deutschen Kameralisten als ordentlicher Professor für Philosophie lehrte und 1761 seine „Rosenschule“ in Camsdorf, eine der ersten Industrieschulen Deutschlands, einrichtete, die auch den Jenenser Studenten als Praktikumsstätte diente ${ }^{96}$. Auch die 1753 in Jena gegründete Physiokratische Gesellschaft nützlicher Wissenschaften gehört in diesen Kontext. Die wirkliche Bedeutung dieser ökonomisch-utilitäres Gedankengut verbreitenden Bewegung für den naturwissenschaftlichen Hochschulunterricht wäre noch im einzelnen zu prüfen; ihre Existenz beweist das Entstehen einer neuen Form von Öffentlichkeit, die in und außerhalb der Universität als potentieller Adressat des chemischen Hochschulunterrichts in Betracht kam und in erster Linie an einer Chemie interessiert war, die nicht bloß auf medizinische Anwendung zielte. In Jena ist dies in der Tat zu beobachten.

Bereits 1728 hatte nämlich Georg Erhard Hamberger, damals noch außerordentlicher Professor der Medizin, parallel zu Teichmeyers Vorlesungen über medizinische Chemie ein eigenes chemisches Kolleg angezeigt, das er 1731 als „Chimia theoretica“ mit zugehörigen Privatübungen fortsetzte und auch dann noch weiterführte, als er 1737 Ordinarius für Mathematik und Naturwissenschaften in der Philosophischen Fakultät wurde. Erst als ihm 1744 zusätzlich die ordentliche medizinische Professur für Botanik, Anatomie und Chirurgie übertragen wurde, hat er sich rein medizinischen und physiologischen Gegenständen zugewendet. Hamberger ist aus seiner skandalösen Kontroverse mit Albrecht von Haller als dogmatischer Iatromechaniker wohlbekannt; chemische Arbeiten hat er nicht publiziert. Eindeutiger der nichtmedizinisch-chemischen Richtung zuzurechnen ist der Wolffianer Laurenz Johann Daniel Suckow, der 1756 in Jena eine ordentliche Professur für Naturlehre erhalten hatte und bis 1787 Privatissima über physische Chemie auf der Grundlage seines Lehrbuches anzeigte ${ }^{97}$. Sein Entwurf einer Physischen Scheidekunst (Frankfurt/Leipzig 1769) zeigt, wie Suckows Chemie beschaffen war: ein teils naturhistorisch, teils nach Operationen und Stoffklassen unterteiltes, philosophisch-rationalistisch überfrachtetes Kompendium, in dem medizinisch-pharmazeutische Aspekte kaum, gewerblich-praktische gelegentlich zur Sprache kommen. Sein Sohn Georg Adolph Suckow, der sich in Jena habilitierte und 1774 als Professor für Chemie und Kameralwissenschaften an die Kameral-Hohe Schule nach Lautern ging, zählt mit seinem auch im Jenaer Hochschulunterricht verwandten Werk Anfangsgründe der ökonomischen und technischen Chemie (Leipzig 1784, ${ }^{2} 1789$ ) zu den Exponenten der Kameralchemie des 18. Jahrhunderts. So war der Boden für die Errichtung einer eigenen chemischen Lehrstelle innerhalb der Philosophischen Fakultät bereitet. Die Verwirklichung lag in den Händen des zuständigen Ministers Goethe, der darin vom Weimarischen Großherzog persönlich unterstützt wurde 98 .

Goethe hatte für die neue Position bereits 1785 den Apothekenprovisor Johann Friedrich August Göttling ausersehen und den wissenschaftlichen Autodidakten auf Staatskosten zwei Jahre zum Studium nach Göttingen und anschließend nach England und Holland geschickt. Zurückgekehrt erhielt Göttling 1789 die Ernennung zum außerordentlichen Professor für Scheidekunst, Arzneikunst und Gewerbskunde in der Philosophischen Fakultät, welche ihn, um der Form zu genügen, rasch zuvor noch zum Doktor der Philosophie promovierte. Bereits im Sommersemester 1789 hielt er Vorlesungen über Experimèntalchemie und Technologie ${ }^{99}$. Doch als Göttling im Winter 1791/92 außerdem 
noch pharmazeutische Chemie ankündigte, stieß er auf den Widerstand der Mediziner. Diese befanden nämlich, daß die Chemie seit jeher zu den Lehrbefugnissen ihrer Fakultät gehört habe und Göttlings Vorgehen die vitalen Interessen dreier medizinischer Dozenten berühre, die bis dahin abwechselnd "Chemie der Arzneimittel“ vorgetragen hatten. Ausführliche Gutachten beider Fakultäten wurden eingeholt, wobei die Philosophische Fakultät die Zugehörigkeit der Chemie zur allgemeinen Naturlehre und die langjährige unangefochtene Lehrtätigkeit Suckows herausstellte. Schließlich einigte man sich darauf, daß beide Fakultäten das Recht erhielten, über allgemeine Chemie zu lesen, die pharmazeutisch-chemischen Vorlesungen aber allein in die Kompetenz der Medizinischen Fakultät fallen sollten und Göttling jeweils um Genehmigung nachsuchen mußte, wenn auch er "Apothekerchymie“ lesen wollte ${ }^{100}$. Damit hatte die Chemie als Teil der allgemeinen und angewandten Naturwissenschaften ihren Platz in der Philosophischen Fakultät behaupten können, wenn auch nur mit einem Extraordinariat, das zwanzig Jahre später in eine ordentliche Professur aufgewertet wurde. Auch war die - dann erst unter Göttlings Nachfolger Johann Wolfgang Döbereiner 1820 vollzogene - Abtrennung der Pharmazie vom chemischen Hochschulfach angebahnt.

Weitere Beispiele für einen auf Dauer erfolgreichen Übertritt in die Philosophische Fakultät bieten die Universitäten Halle (1799), Erlangen (1799), Königsberg (1807), Prag (1812), Gießen (1818), Wien (1838) und Marburg (1841); doch im allgemeinen haben die Hochschulen diesen Schritt erst in der zweiten Hälfte des 19. Jahrhunderts vollzogen.

$x$

Wir haben gesehen, wie sich in der Geschichte des chemischen Hochschulfaches während des 18. Jahrhunderts verschiedene divergierende und miteinander konkurrierende disziplinäre Programme herausbildeten, deren einzelne Richtungen sich weniger hinsichtlich der jeweils verfügbaren chemischen Kenntnisse und Theorien als hinsichtlich der ihnen zugrundeliegenden Leitvorstellungen unterschieden. In diese gingen mentale und kognitive Elemente ebenso ein wie die Besonderheiten des historischen Ortes und konkreten Erfahrungsraumes sowie die auf die Institutionalisierung des eigenen Tuns gerichteten Strategien der Wissenschaftler. Deutlich geworden sind Mechanismen und Triebkräfte des Wissenschaftwandels, soziale und sozialpsychologische Aspekte der Disziplinbildung und nicht zuletzt ein Strukturwandel von Wissenschaft überhaupt, der mit der Auflösung der hierarchischen Schichtung zu einer funktionalen Differenzierung nach Spezialdisziplinen führte, die zu den charakteristischen Merkmalen moderner Wissenschaft gehört.

1 Vgl. hierzu die Ausführungen bei D. von Engelhardt: Zu einer Sozialgeschichte der romantischen Naturforschung. Sudhoffs Archiv 65 (1981), 209-225, wo allerdings an der ontologischen Differenz von "sozialem Hintergrund" und den "substantiellen Voraussetzungen“ festgehalten und nicht konsequent zwischen Sozialgeschichte der Wissenschaft im engeren Sinne und der historischen Wissens- bzw. Wissenschaftssoziologie unterschieden ist.

2 Die Sozialgeschichte der Medizin, deren Gegenstand komplexer und deren Erforschungsstand besser ist als der der Sozialgeschichte der Naturwissenschaften, muß aus den vorliegenden Überlegungen ausgespart bleiben, doch sei verwiesen auf A. Labisch: Zur Sozialgeschichte der Medizin. Methodologische Überlegungen und Forschungsbericht. Archiv für Sozialgeschichte 20 (1980), 431-469.

3 Siehe etwa E. Schmauderer (Hrsg.): Der Chemiker im Wandel der Zeiten. Skizzen zur geschichtlichen Entwicklung des Berufsbildes. Weinheim 1973; B. W. G. Holt: Social Aspects in the Emergence of Chemistry as an Exact Science: The British Chemical Profession. British Journal of Sociology 21 (1970), 181-199; 
K. Hufbauer (a): Social Support for Chemistry in Germany during the Eighteenth Century. How an why did it change? Historical Studies in the Physical Sciences 3 (1971), 205-231; B. H. Gustin: The Emergence of the German Chemical Profession, 1790-1867. Ph.D. dissertation, University of Chicago, Chicago, 1975; L. Burchardt: Professionalisierung oder Berufskonstruktion? Das Beispiel des Chemikers im wilhelminischen Deutschland. Geschichte und Gesellschaft 6 (1980), 326-348; K. Hufbauer (b): The Formation of the German Chemical Community, 1720-1795. Berkeley/Los Angeles/London 1982; R. Bug/G.K. Roberts: Science versus Practice. Chemistry in Victorian Britain. Manchester 1984. Die sozialgeschichtlichen Aspekte der technisch-industriellen Chemie sind in der Forschung bisher sehr vernachlässigt worden; eine frühe Ausnahme bildet die wenig beachtete Arbeit von A. Clow/N. L. Clow: The Chemical Revolution. A Contribution to Social Technology. London 1952.

4 Beispielsweise J. B. Morrel: The Chemist Breeders. The Research Schools of Liebig and Thomas Thomson. Ambix 19 (1972), 1-46 ; G. K. Roberts: The Royal College of Chemistry (1845-1853). A Social History of Chemistry in early-Victorian England. Ph.D. dissertation, The Johns Hopkins University, Baltimore 1973; C. A. Russell/N. G. Coley/G. K. Roberts: Chemists by Profession. The Origins and Rise of The Royal Institute of Chemistry. Milton Keynes 1977.

5 Für einen ersten Versuch, die breite chemische Lehrbuchtradition zu sichten und auch unter sozial- bzw. disziplingeschichtlichen Kriterien anzugehen vgl. die Arbeit von B. Haupt: Deutschsprachige Chemielehrbücher, 1775-1850. Naturwiss. Diss., Marburg 1984.

6 Unter "Chemie“, „chemischem Hochschulfach“ oder "chemischer Wissenschaft" sei im folgenden zweierlei verstanden: einmal der theoretische und praktische Umgang mit stofflichen Vorgängen und Eigenschaften, unabhängig davon, ob die Form dieses Umganges den Kriterien moderner Wissenschaftlichkeit genügen würde; andererseits aber auch die soziale Kategorie „Chemie“, so wie sie jeweils historisch geprägt und benannt war. Zum ersten Aspekt siehe J. Weyer: Die Entwicklung der Chemie zu einer Wissenschaft zwischen 1540 und 1740. Berichte zur Wissenschaftsgeschichte 1 (1978), 113-121.

7 C. Meinel: De praestantia et utilitate Chemiae. Selbstdarstellung einer jungen Disziplin im Spiegel ihres programmatischen Schrifttums. Sudhoffs Archiv 65 (1981), 366-389.

8 Vgl. H.-W. Prahl: Sozialgeschichte des Hochschulwesens. München 1978, bes. S. 29-38; R. Stichweh: Differenzierung der Wissenschaft. Zeitschrift für Soziologie 8 (1979), 82-101.

9 Vgl. auch C. E. Rosenberg: Toward an Ecology of Knowledge. On Discipline, Context and History. In: A. Oleson/J.Voss (Eds.): The Organization of Knowledge in Modern America, 1860-1920. Baltimore 1979, S. 440-455, und bes. S. 443: „It is the discipline that ultimately shapes the scholar's vocational identity. The confraternity of his acknowledged peers defines the scholar's aspirations, sets appropriate problems and provides the intellectual tools with which to address them; finally it is the discipline that rewards intellectual achievement. At the same time his disciplinary identity helps structure the scholar or scientist's relationship to a particular institutional context. His professional life becomes then a compromise defined by the sometimes consistent and sometimes conflicting demands of his discipline and the conditions of his employment".

10 R. E. Kohler: From Medical Chemistry to Biochemistry. The Making of a Biomedical Discipline.(Cambridge Monographs on the History of Medicine) Cambridge/London/New York 1982.

11 Vgl. etwa Johannes Scotus: Annotationes in Marcianum. Hrsg. von C. E. Lutz. Cambridge, Mass. 1939, 60,3: „frequenter inter artem et disciplinam hoc interest, quod quando discitur, disciplina vocatur, quando perfecta in habitu mentis est, ars nuncupatur".

12 Die Herausbildung wissenschaftlicher Disziplinen in der Geschichte. Hrsg. von der Wilhelm-PieckUniversität Rostock. (Rostocker Wissenschaftshistorische Manuskripte, 1) Rostock 1978; vgl. hierin besonders M. Guntau: Zur Herausbildung wissenschaftlicher Disziplinen in der Geschichte (Thesen) (S. 11-24).

$13 \mathrm{Vgl}$. Probleme der Disziplingenese in der Wissenschaftsgeschichte und Wissenschaftsgeschichtsschreibung. Hrsg. von der Wilhelm-Pieck-Universität Rostock. (Rostocker Wissenschaftshistorische Manuskripte, 8) Rostock 1982, und hierin besonders M. Guntau: Gedanken zur Herausbildung wissenschaftlicher Disziplinen in der Geschichte und zu Problemen der Disziplingenese in der Wissenschaftsgeschichtsschreibung (S. 19-49). Vgl dazu auch S. Fahrenbach/U. Köster: Vergleichende Untersuchungen zur Enstehung wissenschaftlicher Disziplinen. Deutsche Zeitschrift für Philosophie 31 (1983), 627-631. Auf diesem Ansatz aufbauend vgl. für die Chemie besonders H. Scholz: Zur Periodisierung des Entstehungsprozesses naturwissenschaftlicher Disziplinen, dargestellt am Beispiel der Entwicklung der Chemie. Deutsche Zeitschrift für Philo. sophie 31 (1983), 89-97.

14 T. S. Kuhn: The Structure of Scientific Revolutions [1962]. (International Encyclopedia of Unified Science, II/2) ${ }^{2}$ Chicago 1970.

15 J. Ben-David: The Scientist's Role in Society. A Comparative Study. Englewood Cliffs 1971. 
16 F. R. Pfetsch/A. Zloczower: Innovation und Widerstände in der Wissenschaft. Beiträge zur Geschichte der deutschen Medizin. (Wissenschaftstheorie, Wissenschaftspolitik, Wissenschaftsplanung, 19) Düsseldorf 1973.

17 P. Weingart: Wissenschaftlicher Wandel als Institutionalisierungsstrategie. In: P. Weingart (Hrsg.): Wissenschaftssoziologie II. Determinanten wissenschaftlicher Entwicklung. (Fischer Athenäum Taschenbücher Sozialwissenschaften, 4008) Frankfurt 1974. Vgl. hierin auch die mittlerweile klassischen Beiträge von J. Ben-David/R. Collins: Soziale Faktoren im Ursprung einer neuen Wissenschaft : Der Fall der Psychologie [1966] (S. 122-152), sowie N. C. Mullins: Die Entwicklung eines wissenschaftlichen Spezialgebiets: die Phagen-Gruppe und die Ursprünge der Molekularbiologie [1972] (S. 184-222).

18 R. Stichweh (a): Ausdifferenzierung der Wissenschaft - Eine Analyse am deutschen Beispiel. (Report Wissenschaftsforschung, 8) Bielefeld 1977; sowie besonders derselbe (b): Zur Entstehung des modernen Systems wissenschaftlicher Disziplinen. Physik in Deutschland, 1740-1890. Frankfurt am Main 1984.

19 N. Luhmann: Gesellschaftsstruktur und Semantik. Studien zur Wissenssoziologie der modernen Gesellschaft. 2 Bde, Frankfurt am Main 1980-1981.

$20 \mathrm{Vgl}$. F. Krafft: Zielgerichtetheit und Zielsetzung in Wissenschaft und Natur. Berichte zur Wissenschaftsge. schichte 5 (1982), 53-74. Zum Entwicklungsbegriff siehe etwa K. Weyand: Entwicklung I. In: J. Ritter (Hrsg.): Historisches Wörterbuch der Philosophie. Bd.2, Basel/Stuttgart 1972, Sp. 550-557.

21 Die Frage nach dem naturwissenschaftlichen Progress fällt damit allerdings aus dem Problemhorizont einer Sozialgeschichte der Wissenschaften heraus; doch sehe ich keinen überzeugenden Weg, wie die Sozialgeschichte der Wissenschaften mit dem ihr eigenen Instrumentarium Kriterien für wissenschaftlichen Erkenntnisfortschritt entwickeln könnte.

22 R. E. Kohler (wie Anm.10).

23 C. E. Rosenberg (wie Anm. 9).

24 Vgl. auch R. W. Home: Out of a Newtonian Straitjacket. Alternative Approaches to Eighteenth-Century Physical Science. In: R. F. Brissenden/J. C. Eade (Eds.): Studies in the Eighteenth Century. Bd 4, Canberra 1979, S. 235-249.

25 Näher ausgeführt in C. Meinel : „Chemia artibus academicis jure inserenda“ - Die Chemie im Streit der Fakultäten. In Vorbereitung.

26 Vgl. hierzu besonders A. G. Debus (a): Science and Education in the Seventeenth Century. The Webster - Ward Debate. (History of Science Library) London/New York 1970 ; derselbe (b): The Chemical Philosophy. Paracelsian Science and Medicine in the Sixteenth and Seventeenth Centuries. 2 Bde, New York 1977, bes. S. 381-531.

27 Vgl. B. T. Moran: Privilege, Communication and Chemiatry. The Hermetic-Alchemical Circle of Moritz of Hessen-Kassel. Ambix 32 (1985), 110-126; hier S. 121.

28 Vgl. dazu H.-H Eulner: Die Entwicklung der medizinischen Spezialfächer an den Universitäten des deutschen Sprachgebietes. (Studien zur Medizingeschichte des 19. Jahrhunderts, 4) Stuttgart 1970, S. 7-8.

29 Für die besonders in England charakteristische Erscheinung solcher „freier“ Dozenten vgl. F. W. Gibbs: Itinerant Lecturers in Natural Philosophy. Ambix 8 (1960), 111-117.

30 Das Phänomen ist typisch für die Übergangsphase von der alten Universitätsverfassung, in der jeder Magister bzw. Doktor die Lehrbefugnis besaß, zur stärker reglementierten Privatdozentur. Sozialgeschichtlich wäre hier, wenn auch auf schwieriger Quellenbasis, noch viel zu tun. Allgemein dazu siehe A. Busch: Die Geschichte des Privatdozenten. Eine soziologische Studie zur großbetrieblichen Entwicklung der deutschen Universitäten. (Göttinger Abhandlungen zur Soziologie, 5), Stuttgart 1959, der allerdings die Zeit vor 1800 nur kurz berührt. Zentral dafür noch immer [J. D. Michaelis:] Raisonnement über die protestantischen Universitäten in Deutschland. Bd 3, Frankfurt/Leipzig 1773, bes. S. 1-63.

31 Vgl. Meinel (wie Anm. 7). Auch Holt (wie Anm. 3), S. 182, weist darauf hin, daß die Herausbildung eines fachlich-sozialen Identitätsgefühls der Disziplinbildung vorausgeht und für ihren Gang instrumentell wird.

32 Vgl. dazu A. Thackray: Atoms and Powers. An Essay on Newtonian Matter-Theory and the Development of Chemistry. (Harvard Monographs in the History of Science). Cambridge, Mass. 1970.

33 C. Meinel: Reine und angewandte Chemie. Die Entstehung einer neuen Wissenschaftskonzeption in der Chemie der Aufklärung. Berichte zur Wissenschaftsgeschichte 8 (1985), 25-45.

$34 \mathrm{Vgl}$. C. Meinel (wie Anm. 25).

35 Frühe Vorstöße in diese Richtung unternahmen die Universitäten Würzburg (1734) und Königsberg 1737.

36 Vgl. C. Meinel (wie Anm. 33).

37 Vgl. K. Hufbauer: Chemistry's Enlightened Audience. Studies on Voltaire and the Eighteenth Century 153 (1976), S. 1069-1086. 
38 Vgl. F. Eulen: Die Technologie als ökonomische und technische Wissenschaft an deutschen Universitäten des 18. Jahrhunderts. Technikgeschichte 36 (1969), 245-256.

39 Vgl. auch R. Stichweh (wie Anm, 18/b), S. 57.

40 Nach dem Vorbild von Uppsala (1750) hatten die Universitäten Lund (1758), Åbo (1761) und Christiania (1839) ihre neuerrichteten Chemieprofessuren nicht, wie es in anderen Ländern üblich war, der Medizin, sondern innerhalb der Philosophischen Fakultät den ökonomischen Wissenschaften angegliedert.

41 Hier besonders die Bergakademien in Freiberg/Sachsen (Hochschulcharakter seit 1765) und Schemnitz (1770), die Bergakademie Berlin (1770) und die Pariser École des Mines (1783). Eine wissenschaftshistorische Untersuchung dieses auch sozialgeschichtlich bemerkenswerten Hochschultyps steht noch aus.

42 So z. B. Christian Ehregott Gellert, 1765 Professor für Chemie und Metallurgie an der Bergakademie Freiberg; Valentin Rose, 1770 Professor für Chemie an der Berliner Bergakademie; Thaddäus Peithner, 1772 Professor für Chemie und Montanwissenschaften an der Bergakademie Schemnitz.

43 Im Sinne ihres Begründers Johann Beckmann die Wissenschaft von der Gewinnung und Veredelung der Naturprodukte, mithin ein Fach, das auf eine chemisch fundierte Stoff- und Warenkunde nicht verzichten konnte. Vgl. dazu auch U. Troitzsch: Ansätze technologischen Denkens bei den Kameralisten des 17. und 18. Jahrhunderts. (Schriften zur Wirtschafts- und Sozialgeschichte, 5) Berlin 1966, bes. S. 154-161.

44 Auch im Falle der Kameralwissenschaften war die Zuordnung zum traditionellen Fakultätenschema strittig; als typische „Brotwissenschaft” schien sie nicht in die Philosophische Fakultät zu passen, vom Gegenstand her war sie dem festgefügten Fächerkanon der Juristischen Fakultät fremd, so daß sich die strukturell bemerkenswerte, wenn auch universitätsgeschichtlich bisher kaum untersuchte Bildung einer eigenen, fünften Fakultät bzw. die Einrichtung fakultätsübergreifender Institutionen, denen Professoren unterschiedlicher Fakultäten angehörten, als Ausweg anbot. So finden sich derartige Chemieprofessuren 1760 an der neugegründeten Universität Bützow, 1774 an der Cameral-Hohen Schule zu Lautern, 1784 an ihrer Nachfolgerin, der Staatswirtschafts-Hohen Schule zu Heidelberg und im gleichen Jahr an der Universität Dillingen; darüberhinaus an der Kameralwissenschaftlichen Fakultät der Universität Gießen (1777-1785), als Professur für Ökonomie und Kameralchemie an der Universität Wien (1838) sowie in Gestalt der Zugehörigkeit des Chemieprofessors, der hauptamtlich Mitglied der Medizinischen Fakultät war, zum Staatswirtschaftlichen Institut an der Universität Marburg (1789-1844) und zur Kameralwissenschaftlichen Fakultät der Universität Mainz (1784-1798).

45 Bemerkenwert ist, daß im umfangreichen Werk Lavoisiers neben den Beiträgen zur Theorie und Nomenklatur der Chemie zwar physiologische Fragen eine Rolle spielen, die Pharmazie aber vollkommen fehlt. Quantitativ dominieren die Arbeiten zur gewerblich-industriellen Chemie, Agrikultur und „Ökonomie“. Das gleiche gilt für andere Vertreter dieser Richtung wie Gay-Lussac, wohingegen Fourcroy, der eine medizinische Ausbildung durchlaufen hatte, immer wieder auch rein pharmazeutische Arbeiten publiziert hat.

46 Zunächst in Österreich 1804, vgl. K. Ganzinger: Gerard van Swieten und die Pharmazie. Österreichische Apotheker-Zeitung 26 (1972), 452f.; danach auch in Bayern durch das „Organische Edikt über das Medicinalwesen im Königreich Bayern“ vom 8. September 1808, dessen Bestimmungen über ein Pflichtstudium der Pharmazeuten Preußen 1825 übernahm.

47 Vgl. R. S. Turner (a): The Growth of Professorial Research in Prussia, 1818 to 1848 - Causes and Context. Historical Studies in the Physical Sciences 3 (1971), 137-182; derselbe (b): University Reformers and Professorial Scholarship in Germany, 1760-1806. In: L. Stones (Ed.): The University in Society. Bd 2, Princeton, N. J. 1974, S. 495-531.

48 Vgl. B. Gustin: The Emergence of the German Chemical Profession, 1790-1867. Ph.D. dissertation, The University of Chicago, Chicago 1975; E. Hickel: Der Apothekerberuf als Keimzelle naturwissenschaftlicher Berufe in Deutschland. Medizinhistorisches Journal 13 (1978), 259-276. Zur Bedeutung der pharmazeutischen Reformbewegung und insbesondere zur Rolle der pharmazeutischen Privatinstitute für die Transformation des chemischen Hochschulfaches vgl. D. Pohl: Zur Geschichte der pharmazeutischen Privatinstitute in Deutschland von 1779 bis 1873. Naturwiss. Diss., Marburg 1972. Weitere Hinweise bei C. Meinel (wie Anm. 25).

49 Vgl. J. Valentin: Die Entwicklung der pharmazeutischen Chemie an der Ernst Moritz Arndt-Universität in Greifswald. In: Festschrift zur 500-Jahrfeier der Universität Greifswald. Hrsg. von der Ernst Moritz Arndt-Universität Greifswald. Bd 2, Greifswald 1956, S. 468-482.

50 I. Seth: Die Universität Greifswald und ihre Stellung in der schwedischen Kulturpolitik, 1637-1815. Berlin 1956.

51 E. T. Nauk: die Zahl der Medizinstudenten der deutschen Hochschulen im 14.-18. Jahrhundert. Sudhoffs Archiv 38 (1954), 175-186; für die Zeit C. E. Weigels sind diese Angaben, wie mir Dr. Christoph Friedrich aus Greifswald freundlicherweise mitteilte, revisionsbedürftig. 
52 Dazu ist es trotz zahlloser Anträge nicht gekommen.

$53 \mathrm{Vgl}$. H. Schröder: Zur politischen Geschichte der Ernst Moritz Arndt-Universität Greifswald. In: Festschrift (wie Anm. 49), Bd 1, S. 53-155.

54 J. D. von Reichenbach: Patriotische Beyträge zur Kenntnis und Aufnahme des schwedischen Pommerns. 5. Stück, Greifswald 1786, S. 180-181. Die Kritik provozierte eine Gegendarstellung aus der Feder des Greifswalder Chemieprofessors C. E. Weigel : Ueber die Academie zu Greifswald, gegen Hrn. Cammerrath von Reichenbach. Stralsund 1787. Dieser erwiderte sie im 7. Stück seiner Patriotischen Beyträge. Stralsund 1787.

55 Offenbar in direkter Wirkung der Antrittsrede vom C. E. Weigel (a): Vom Nutzen der Chemie insbesondere in Absicht auf Pommern betrachtet. Greifswald 1774; vgl. derselbe (b). Der Einfluß Chemischer Kenntnisse in die Oekonomie besonders des Schwedischen Pommerns. Greifswald 1775, S. 4.

56 E. M. Arndt: Erinnerungen aus dem äußeren Leben. Rudolstadt 1953, S. 89-90.

57 C. Friedrich/H. Langer: Christian Ehrenfried von Weigel. Ein bedeutender Naturwissenschaftler an der Universität Greifswald. Greifswald-Stralsunder Jabrbuch 13/14 (1982), 102-115; C. Friedrich/H. Langer/H.-J. Seidlein: Beiträge zur Geschichte der pharmazeutischen Wissenschaft III: Christian Ehrenfried von Weigel - Seine Bedeutung für die Entwicklung der pharmazeutischen Wissenschaft. Die Pharmazie 37 (1982), 290-293, 446-450, 514-517.

58 Vgl. C. Meinel (wie Anm. 33), bes. S. 37.

59 Vgl. C. Meinel (wie Anm.33), S. 32-34.

60 K. Hufbauer (wie Anm. 3/b), S. 246-248, gibt hier ein merkwürdig verzerrtes Bild. Vgl. noch W. Kaiser: Die Naturwissenschaften im halleschen Lehrprogramm des 18. Jahrhunderts. In: W. Kaiser/B. Thaler (Hrsgg.): Johann Andreas Segner (1704-1777) und seine Zeit. (Wissenschaftliche Beiträge der MartinLuther-Universität Halle-Wittenberg, 1977/36 〈T 20〉) Halle 1977, S. 57- 93.

$61 \mathrm{Vgl}$. W. Kaiser /K.-H. Krosch: Die Statuten der Medizinischen Fakultät im 18. Jahrhundert. In: 250 Jahre Collegium Clinicum Halense, 1717-1967. Beiträge zur Geschichte der Medizinischen Fakultät der Universität Halle. (Wissenschaftliche Beiträge der Martin-Luther-Universität Halle-Wittenberg, 1967/3〈R 2〉) Halle 1967, S. 77-103.

62 Vgl. W. Kaiser/ H. Krosch: Extraordinarien und Doctores legentes. Wissenschaftliche Zeitschrift der MartinLuther-Universität Halle-Wittenberg, Math.-naturwiss. Reibe 14 (1965), 357-390.

$63 \mathrm{Vgl}$. W. Kaiser/K.-H. Krosch: Zur Geschichte der Medizinischen Fakultät der Universität Halle im 18. Jahrhundert (I). Wissenschaftliche Zeitschrift der Martin-Luther-Universität Halle-Wittenberg, Math. naturwiss. Reibe 13 (1964), 141-180.

64 Vgl. W. J. G. Karsten (a): Vom eigenthümlichen Gebiet der Naturlehre. In: Derselbe (b): Physischchemische Abhandlungen. Halle 1786-1787, Bd 1, S. 97-199, Bd 2, S. 1-154.

65 Die Magisterpromotion war nahezu außer Gebrauch gekommen, vgl. J. D. Michaelis: Raisonnement über die protestantischen Universitäten in Deutschland. Bd 4, Frankfurt/Leipzig 1776, S. 109-112.

66 Gren an den König, 1788 Aug. 2, zitiert nach W. Kaiser/K.-H. Krosch (wie Anm. 63), S. 171.

67 Nach dem Bericht des Kanzlers an den König, 1788 Sept. 15, ebd., S. 171.

$68 \mathrm{Vgl}$. auch E. Ströker: Theoriewandel in der Wissenschaftsgeschichte. Chemie im 18. Jahrhundert. Frankfurt am Main 1982, bes. S. 289-290.

69 Vgl. R. Möller: Beiträge zur Biographie A. N. Scherers. NTM Schriftenreibe für Geschichte der Naturwissenschaften, Technik und Medizin 2/6 (1965), 37-55.

70 Die Pharmazie war damit ganz aus dem Lehrplan verschwunden und erst wieder 1810 mit einer außerordentlichen Professur für Johann Friedrich Düffer in der Medizinischen Fakultät vertreten.

71 Auch die in Halle immerhin seit 1727 mit einem eigenen Lehrstuhl in der Philosophischen Fakultät vertretene, wenn auch nie sehr glücklich (zuletzt mit Johann Christian Rüdiger) besetzte Kameralistik scheint keinen Einfluß auf die Chemie genommen zu haben.

72 Vgl. hierzu G.-A. Ganss: Geschichte der pharmazeutischen Chemie an der Universität Göttingen. Naturwiss. Diss. Göttingen 1937; G. von Selle: Die Georg-August-Universität zu Göttingen, 1737-1937. Göttingen 1937; J. S. Pütter: Versuch einer academischen Gelehrten-Geschichte von der Georg-AugustusUniversität zu Göttingen. 2 Bde, Göttingen 1765-1788.

73 Vgl. G. von Selle (wie Anm. 72), S. 20-28.

74 Vgl. Paul Gottlob von Werhof: Erfordertes Gutachten wegen einer medicinischen Facultät. In : E. F. Rössler (Hrsg.): Die Gründung der Universität Göttingen. Entwürfe, Berichte und Briefe der Zeitgenossen. Göttingen 1855, S. 298-304; hier S. 298-300.

75 Bemerkungen über Johann Jacob Mosers Rede, wie Universitäten, besonders in der juristischen Facultät, in Aufnahme zu bringen und darinn zu erhalten - aus den Papieren eines verstorbenen Staatsministers und Universitäts-Curators. In: E. F. Rössler (wie Anm. 74), S. 468-486; hier S. 472. 
76 Vgl. W. Ebel (Hrsg.): Die Privilegien und ältesten Statuten der Georg-August-Universität zu Göttingen. Göttingen 1961, S. 152-173. Auch der Göttinger Orientalist J. D. Michaelis machte sich in seinem Raisonnement über die protestantischen Universitäten in Deutschland (Frankfurt/Leipzig 1770-1776) zum Fürsprecher einer ungehinderten Lehr- und Aemulationsfreiheit.

77 Vgl. G. Beer: Der Versuch Johann Christoph Cron's zur Errichtung eines ersten Chemischen Laboratoriums an der Universität Göttingen im Jahre 1735. Göttinger Jabrbuch 28 (1980), 97-108.

78 Segner hat nie eigentlich medizinische Themen bearbeitet, sondern ist als Mathematiker, Astronom und Mechaniker bekannt; die Ernennung verdankte er überwiegend dem Umstand, daß er Schüler des Helmstedter Chirurgen Lorenz Heister war; vgl. auch W. Kaiser/B. Thaler (wie Anm. 60).

79 Vgl. H. Schimank: Zur Geschichte der Physik an der Universität Göttingen vor Wilhelm Weber, 1734-1830. Rete 2 (1973/75), 207-252; hier bes. S. 223-235.

80 Vgl. W. Ebel (a): Entwicklungsgeschichte des Göttinger Privatdozenten. In: Derselbe (b): Memorabilia Gottingensia. Elf Studien zur Sozialgeschichte der Universität. Göttingen 1969, S. 57-72, mit dem Nachweis, daß die moderne Form der Habilitation ihren Ursprung in Göttingen und nicht in Berlin hat.

81 Hofrath J. D. Gruber an Gerl. Adolf von Münchhausen über den Zustand und die Blüthe der deutschen Universitäten. In: E. F. Rössler (wie Anm. 74), S. 458-467; hier S. 462.

82 Geheimer Rat an Kgl. Ministerium, Hannover, 1774 Nov 16, Promemoria, protokollweise beschrieben und paraphiert, Universitätsarchiv Göttingen, Personalakten Med. Fakultät, 2) Specialia, a) Lehrer, Az. $4 / \mathrm{IV}$ b 21.

83 Wie Anm. 82

84 Gmelin an Hann. Räte, Tübingen, 1774 Dez 1, eigenh., ohne Präsentatvermerk; Georg III an Hann. Räte, behänd. Reskript, St. James, 1775 Jan 6, Universitätsarchiv Göttingen, Personalakten Med. Fakultät, 2) Specialia, a) Lehrer, Az. 4/IV b 21.

85 Geh. Räte an Gmelin, Note, Hannover, 1778 Nov 26, Universitätsarchiv Göttingen, Personalakten Med. Fakultät, 2) Specialia, a) Lehrer, Az. 4/IV b 21.

86 J. S. Pütter (wie Anm. 72), Bd 2, S. 146-148.

87 J. S. Pütter (wie Anm. 72), Bd 2, S. 330-331.

88 Besonders J. G. Wallerius: Åkerbrukets chemiska Grunder. Stockholm 1761; lat. als: Agriculturae fundamenta chemica. Stockholm 1761; deutsch unter dem Titel: Chemische Grundsätze des Feldbaus. Berlin 1764.

89 Für die hier nicht näher verfolgten Bezichungen der Chemie zur Naturkunde bzw. Naturgeschichte und entstehenden Hochschulphysik siehe R. Stichweh (wie Anm. 18/b), bes. S. 99-115.

90 Vgl. B. Haupt (wie Anm. 5).

91 J. C. P. Erxleben: Anfangsgründe der Chemie. Göttingen 1775, Vorrede; hier zitiert nach der zweiten, von J. C. Wiegleb besorgten Auflage, Göttingen 1793, S. IV.

92 Lichtenbergs Beziehung zur Chemie ist kaum untersucht; vgl. aber P. Hahn: Georg Christoph Lichtenberg und die exakten Wissenschaften. Materialien zu seiner Biographie. (Vorarbeiten zur Geschichte der Göttinger Universität und Bibliothek, 4) Göttingen 1927, S. 52-71; D. Goetz: Georg Christoph Lichtenberg. (Biographien hervorragender Naturwissenschaftler, Techniker und Mediziner, 49) Leipzig 1980, S. 52-55.

93 Davon hat bisher fast nur die medizinische Richtung eine eingehende Darstellung erfahren; vgl. E. Giese/ B. von Hagen: Geschichte der Medizinischen Fakultät der Friedrich-Schiller-Universität Jena. Jena 1958. Die Darstellung bei K. Hufbauer (wie Anm. 3/b), S. 252-253, ergibt auch hier ein verzerrtes Bild. Allgemein vgl. noch S. Schmidt (Hrsg.): Alma Mater Jenensis. Geschichte der Universität Jena. Weimar 1983.

94 Vgl. F. Chemnitius: Die Chemie in Jena von Rolfinck bis Knorr, 1629-1921. Jena 1929.

95 Teichmeyers Jenaer Werdegang ist ein Paradebeispiel für das Aufrücken nach Anciennität: von der Professur für Experimentalphysik in der Philosophischen Fakultät (1717) zu der 1719 ein Extraordinariat in der Medizinischen Fakultät hinzukam, auf die vierte (1722), dritte (1727) und schließlich zweite (1731) medizinische Professur.

96 S. Schmidt (wie Anm. 93), S. 121-123.

97 Nach Ausweis der Vorlesungsverzeichnisse erstmals 1776, doch heißt es im Vorwort seines Entwurfs einer Physischen Scheidekunst von 1769, Nachschriften dieses Vorlesungstextes seien bereits seit "vielen Jahren“ kursiert.

98 Vgl. A. Gutbier: Goethe, Großherzog Carl August und die Chemie in Jena. (Jenaer Akademische Reden, 2) Jena 1926; H. Döbling: Die Chemie in Jena zur Goethezeit. (Zeitschrift des Vereins für Thüringische Geschichte und Altertumskunde, N. F., Beiheft 13) Jena 1928. 
99 Letztere anhand von G. F. Lamprecht: Lehrbuch der Technologie oder Anleitung zur Kenntniß der Handwerker. Halle 1787.

100 Vgl. H. Döbling (wie Anm.98), S. 2-6.

Anschrift des Verfassers: Dr. Christoph Meinel, Universität Hamburg, Institut für Geschichte der Naturwissenschaften, Mathematik und Technik, Bundesstraße 55, D-2000 Hamburg 13

\section{Rezension}

Exempla historica - Epochen der Weltgeschichte in Biographien (in 70 Bänden). Frankfurt am Main: Fischer Taschenbuch Verlag $1984 \mathrm{ff}$. Kartoniert, pro Band DM 12,80 oder 14,80 (ca. 220 bis 300 Seiten).

Bei den Bänden dieser Reihe handelt es sich um einen Wiederabdruck der Biographien, die unter der verantwortlichen Herausgeberschaft von Kurt Faßmann in den Jahren 1970-1979 in zwölf Bänden unter dem Titel Die Großen der Weltgeschichte im Kindler Verlag Zürich erschienen. Der Wiederabdruck der Texte wurde von Marion Schmid betreut, die auch die einzelnen Autoren um Ergänzungen und Korrekturen der bibliographischen Angaben gebeten hatte - die bei den ersten Bänden dann doch nicht in den Druck aufgenommen wurden. Später fanden dagegen selbst kleinere Textkorrekturen Berücksichtigung. Die Biographien sind neu zu Bänden von 200-300 Seiten gruppiert worden, die Abbildungen wurden auf wenige, zum Textverständnis erforderliche reduziert. Die bibliographischen Angaben sind am Ende eines Bandes jeweils zusammengafaßt.

Von den Bänden, in denen Biographien von Wissenschaftlern zusammengefaßt sind, erschienen bisher im Epochenabschnitt „Von den frühen Hochkulturen bis zum Hellenismus“ die Bände 4 (219 SS., 1985; DM 12,80) und 5 (245 SS., 1985; DM 14,80) Griechische Philosophen: Anaximandros (F. Krafft), Heraklit (H. H. Holz), Anaxagoras und Empedokles (F. Krafft), Sokrates (E. Schmalzriedt), Demokrit (H. H. Holz), Platon (E. Schmalzriedt), Aristoteles (O. Gigon) und Epikur (O. Gigon) bzw. Forscher und Gelebrte: Imhotep (E. H. Fischer), Ni-Anch-Sachmet (G. Harig), Pythagoras (H. Freudenthal), Herodot (K. von Fritz), Thukydides (K. von Fritz), Hippokrates (H. Schipperges), Euklid (H. Freudenthal), Archimedes (F. Krafft), Hipparch (F. Hinderer); im Epochenabschnitt „Römisches Imperium und frühes Mittelalter" Band 10:
Forscher und Gelebrte (237 SS., 1985; DM 14,80) mit Plinius dem Älteren (E. H. Warmington), Heron von Alexandria (F. Krafft; S. 41-101 mit den wichtigsten Abbildungen zur heronischen Technik und Mathematik), Tacitus (J. von Stackelberg), Ptolemäus (F. Krafft; S. 123-188 mit den wichtigsten Abbildungen zur ptolemäischen Astronomie und Geographie), Galenos (H. Schipperges), Hua T'uo (M. Porkert); sowie im Epochenabschnitt „Die Konstituierung der neuzeitlichen Welt" Band 27 Naturwissenschaftler und Mathematiker (296 SS., 1984; DM 12,80): Gerhard Mercator (E. Bernleithner), André Vésale (G. Rath), Conrad Gessner (H. Heiliger), François Viète (I. Schneider), Tycho Brahe (F. Krafft), Galileo Galilei (P. Jordan), Johannes Kepler (W. Gerlach), Otto von Guericke (F. Krafft), Christian Huygens (G. Franke).

Die Biographien sind unterschiedlich konzipiert, vom wissenschaftlich fundierten Essay bis zur wissenschaftlichen Originalabhandlung, sind aber als Einstieg in das Leben und Werk der betreffenden Persönlichkeit allemal geeignet. Leider scheint der Velag das Erscheinen mitten in der Reihe eingestellt zu haben, so daß man für die Biographien aus der Zeit ab dem späten 17. Jahrhundert wieder auf die (unredigierte) Originalausgabe zurückgreifen muß, die sich nur in den Bibliotheken findet. Es wäre sehr zu wünschen, daß der Verlag sein Vorhaben weiterführt, damit die Biographien dieser Reihe weiterhin auch Studenten und Schülern zu einem erschwinglichen Preis zugänglich gemacht werden.

Fritz Krafft, Mainz 